\title{
FINANCIAL MARKETS WITH A LARGE TRADER ${ }^{1}$
}

\author{
By TILMANN BLÜMMEL ${ }^{2}$ AND THORSTEN RHEINLÄNDER \\ University of Vienna and Vienna University of Technology
}

We construct a large trader model using tools from nonlinear stochastic integration theory and an impact function. It encompasses many well-known models from the literature. In particular, the model allows price changes to depend on the size as well as on the speed and timing of the large trader's transactions. Moreover, a volume impact limit order book can be studied in this framework. Relaxing a condition about existence of a universal martingale measure governing all resulting small trader models, we can show absence of arbitrage for the small trader under mild conditions. Furthermore, a case study on utility maximization from terminal wealth highlights new phenomena that can arise in our framework. Finally, an outlook on further research provides insights on (no) arbitrage opportunities for the large trader and how different levels of information may affect our analysis.

\section{CONTENTS}

1. Introduction . . . . . . . . . . . . . . . . . . . . . . 3736

2. Basic setting and examples for simple large trader strategies . . . . . . . . . . . . . 3740

2.1. Primal family and the price process for simple large trader strategies . . . . . . . . . 3740

2.2. Regularity conditions on the primal family . . . . . . . . . . . . . . . . 3743

2.3. Examples . . . . . . . . . . . . . . . . . . . . . . . . . 3745

2.4. The large trader real wealth process for simple strategies . . . . . . . . . . . . . 3746

3. Price process and real wealth process for dynamic large trader strategies . . . . . . . . . . . 3749

3.1. The price process for dynamic strategies . . . . . . . . . . . . . . . . . . . . 3749

3.2. The large trader real wealth process for dynamic strategies and a critical discussion of

Assumptions I and II . . . . . . . . . . . . . . . . . . . . . . 3752

4. No arbitrage condition for dynamic strategies under Assumption II . . . . . . . . . . 3755

5. Utility maximization: Description of new phenomena arising in a large trader setting . . . . 3760

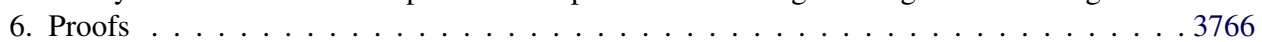

7. Outlook on further research . . . . . . . . . . . . . . . . . . . . . . 3771

7.1. Price process for dynamic strategies and limit order book . . . . . . . . . . . . . 3772

7.2. (No) arbitrage opportunities for the large trader . . . . . . . . . . . . . . . . . . . 3773

7.3. Different levels of information . . . . . . . . . . . . . . . . . . . . 3774

7.3.1. Different levels of information and arbitrage opportunities for the small

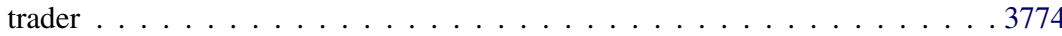

Received December 2015; revised November 2016.

${ }^{1}$ This paper is based on the Ph.D. thesis of the first author as well as an earlier version by the second author and Jan Kallsen.

${ }^{2}$ Supported by the Austrian Science Fund (FWF) Grant P25815.

MSC2010 subject classifications. $60 \mathrm{H} 05,91 \mathrm{G} 10$.

Key words and phrases. Large trader, limit order book, no arbitrage, nonlinear stochastic integration, portfolio optimization. 
7.3.2. Different levels of information and (no) arbitrage opportunities for the large trader . . . . . . . . . . . . . . . . . . . . . . . . . . . . . . . . . . . . . . . . . Appendix A: Strong nonlinear integrators and the nonlinear stochastic integral . . . . . . . 3775

A.1. Simple strategies . . . . . . . . . . . . . . . . . . . . . . 3775

A.2. Semimartingale topology . . . . . . . . . . . . . . . . . . . . . . 3777

A.3. Strong nonlinear integrators . . . . . . . . . . . . . . . . . . . . . . . . . . . 3779

A.4. Nonlinear stochastic integral . . . . . . . . . . . . . . . . . . . . . . . . . . . 3780

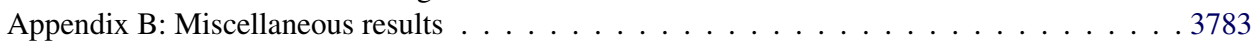

Acknowledgements . . . . . . . . . . . . . . . . . . . . . . 3784

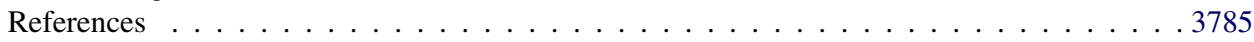

1. Introduction. In mathematical finance, the "competitive market paradigm" is a widely spread and yet unrealistic assumption. It claims that all agents can buy or sell an arbitrary amount of shares without changing the price of the asset. Here, our concern is to drop this paradigm and to consider a large investor who influences prices by means of her transactions.

The "mechanics" behind the impact of the large trader's transactions on the price might have different causes and may appear in many different ways. On one hand, the changes of the price can depend on the size, the speed or the timing of the large trader's transactions. On the other hand, the effects of the transactions on the price can be temporary, permanent or abrupt in the beginning and fading away over time. The last phenomenon is usually referred to as resilience. The model of Almgren and Chriss $[2,3]$ is an example for a large trader model with temporary and permanent price impact, the model of, for example, Gatheral [16] captures the resilience-type of price impact. While both models describe the impact of the large trader's strategy on the price process in a rather direct way as a function of the strategy and its derivative, more recent models use a more indirect way to describe the impact. One example is the volume impact model of Alfonsi and Schied [1], where the authors describe the volume impact of the large trader strategy on the limit order book. Here, the actual price impact is derived from the relation between volume impact and price impact, which is characterized by the shape function of the limit order book.

The "consequences" of the large trader's impact on the price process are farreaching and might affect price takers as well as the large trader herself. First, the large trader faces an additional risk due to her price impact. In the market microstructure literature (see, e.g., Kyle [26]), this risk is referred to as liquidity risk. Simply put, it is the risk a large trader faces due to the timing, the speed and the size of her transactions. Second, the large trader might be able to use her influence on the price process to generate an arbitrage. Furthermore, the turbulences caused by the large trader's actions might also lead to arbitrage opportunities for the small trader.

Aside from the impact of the large trader's transactions on the price and its consequences, the probably most important question is the following: "What is the 
large trader's motivation to trade?" A typical example would be to find an optimal liquidation strategy; see, for example, Schied and co-authors [17, 31-33]. Usually, the optimality criterion is tied to a utility maximization problem or a liquidity cost minimization problem. In this way, the optimization problems constitute a mathematical formulation reflecting the goals of the large trader. Another motivation for the large trader might be the realisation of arbitrage opportunities.

The papers of Çetin, Jarrow and Protter [10] and Bank and Baum [4] are to our knowledge the first that embed the large trader problematic into a sophisticated random field based stochastic model. Using different approaches, both models capture several of the phenomena addressed above. While Çetin, Jarrow and Protter [10] postulate the existence of a stochastic supply curve governing the transactions of the large trader, Bank and Baum [4] model the prices themselves using a random field $(S(\vartheta, \cdot))_{\vartheta \in \mathbb{R}}$ indexed by the large trader's constant position $\vartheta$ in the asset. For a dynamic large trader strategy $\theta$, Bank and Baum [4] model the evolution of the price as $P_{\mathrm{BB}}^{\theta}:=S(\theta \cdot, \cdot)$. As a consequence, any change of the position causes an instantaneous reaction of the price process. From a mathematical point of view, the resulting value process of the large investor has to be modelled by a nonlinear stochastic integral. This is due to the fact that the integrator is affected by the strategy of the large trader. Bank and Baum [4] have chosen the Kunita integral [25] for this purpose. Amongst others, Bank and Baum [4] prove the absence of arbitrage for the large trader in their model and discuss utility maximization from terminal wealth. However, all these results are achieved under the crucial assumption that there exists a universal local martingale measure for all primitive price processes constituting the random field. Moreover, due to the definition of $P_{\mathrm{BB}}^{\theta}$, the impact of the large trader on the price process only depends on the size of her position. Hence, the models of Almgren and Chriss [2, 3] and Gatheral [16] are in general not included in the model class of Bank and Baum [4] as their price processes rather depend on the speed of the large trader's transaction than on its size.

So far we have seen that various authors considered a variety of questions related to the presence of a large trader in a financial market. Besides, the technical frameworks in which the answers are embedded are as diverse as the questions themselves. Here, we present a framework in which the models mentioned above can be embedded. Due to the high level of complexity concerning the different phenomena and their interaction in a market with a large trader, our main purpose is to present a modular approach to the large trader problematic. We focus on the core modules, that is, the definition of the price process affected by a large trader and her real wealth process.

Inspired by the work of Bank and Baum [4], the price process affected by a large trader is provided by a primal family $(S(\vartheta, \cdot))_{\vartheta \in \mathbb{R}^{d}}$ of continuous, $\mathbb{R}$-valued semimartingales which, together with an impact function $F$ and a large trader strategy $\theta$, constitutes the price process $P^{\theta}$. For a simple strategy $\theta$, the price process $P^{\theta}$ is then modelled as the elementary nonlinear stochastic integral of 
$F(\theta)$ w.r.t. the random field $(S(\vartheta, \cdot))_{\vartheta \in \mathbb{R}^{d}}$. For nonsimple strategies, $P^{\theta}$ is defined by using a limit procedure similar to the definition of a strong nonlinear integrator in the sense of Carmona and Nualart [9]. Not only does this approach allow us to capture the different types of price impacts mentioned above, it also enables us to ease ([4], Assumption 3, of Bank and Baum) postulating the existence of a universal equivalent local martingale measure for the whole primal family. Throughout the paper, we work under the standing Assumption I which ensures that the price process affected by an arbitrary simple large trader strategy is a semimartingale. Additionally, for any simple large trader strategy $\theta$, it claims the existence of an equivalent local martingale measure for the price process $P^{\theta}$. Thereby, the resulting price process serves as a reasonable price process for a frictionless small trader market model. Note that this assumption is similar to the assumption in the discrete time framework by Jarrow [20]. Besides Assumption I, we also introduce a stronger Assumption II. It claims that the random field $(S(\vartheta, \cdot))_{\vartheta \in \mathbb{R}^{d}}$ is a strong nonlinear integrator in the sense of Carmona and Nualart [9], and that the impact function $F$ is continuous. Under this mild regularity assumption, it turns out that the existence of equivalent local martingale measures for each element of the primal family $(S(\vartheta, \cdot))_{\vartheta \in \mathbb{R}^{d}}$ is sufficient for Assumption I to hold.

Even more challenging than the definition of the affected price process $P^{\theta}$ is the definition of the real wealth process. This is due to the fact that the book value of a strategy usually deviates from its realizable wealth, which is given by the real wealth process. We propose a definition being a mixture of the definitions given in Bank and Baum [4] and Çetin, Jarrow and Protter [10]. Similar to both, we end up with a representation

$$
V(\theta)=V_{0}^{\theta}+\int \theta d P^{\theta}-C(\theta)
$$

of the real wealth process $V(\theta)$, where $C(\theta)$ represents costs due to liquidity risks and $\int \theta d P^{\theta}$ reflects the accumulated gains and losses due to (impact) trading. This representation becomes even more intuitive if $P^{\theta}$ is the sum of an unperturbed price $\tilde{P}$ and a perturbed price $I^{\theta}$. In this case,

$$
V(\theta)=V_{0}^{\theta}+\int \theta d \tilde{P}+\int \theta d I^{\theta}-C(\theta)
$$

where $\int \theta d \tilde{P}$ can be interpreted as gains and losses due to exogenous shocks while $\int \theta d I^{\theta}$ are gains and losses due to the large trader's price impact. Hence, given (1), the process

$$
\int \theta d I^{\theta}-C(\theta)
$$

can be seen as the overall large trader risk due to illiquidity and price impact. 
Apart from these two modules related to the presence of a large trader, we are interested in other modules as well. These modules are closely related to the motivation behind the large trader's actions, for example, utility maximization from terminal wealth, optimal liquidation of the large trader's portfolio and (no) arbitrage considerations for the large trader. In all of these problems, the large trader's real wealth process and its structure, that is, the impact of $\theta$ on $P^{\theta}$ and the choice of the cost function $C$, play a major role in the analysis. These considerations directly lead back to the core modules as the motivation of the large trader determines the set of large trader strategies for which we have to provide a definition for $P^{\theta}$ and $V(\theta)$. On one hand, from an economic and functional point of view, the subset of simple large trader strategies should always be contained in the analysis. This is reflected in the standing Assumption I. On the other hand, any further technical assumption ensuring the existence of $P^{\theta}$ and $V(\theta)$ for a large class of strategies should be postulated with regard to an economic motivation. For instance, Assumption II enables us to define $P^{\theta}$ for arbitrary càglàd strategies and reduces questions regarding the existence of $V(\theta)$ to an analysis regarding the existence of $C(\theta)$. The economic motivation behind Assumption II is to extend the analysis of (no) arbitrage opportunities for the small trader to prices $P^{\theta}$, driven by general large trader strategies.

Besides the latter and a critical discussion of Assumptions I and II, we provide a case study on utility maximization from terminal wealth for a simple underlying primal family. Despite its plain structure, it illustrates various phenomena which may occur when considering utility maximization from terminal wealth in our setting. In particular, we single out a simple condition which decides whether we are in a stable situation, or whether we encounter an alternative run-away effect, which leads to ultimate destabilisation of the market.

Although the case study on utility maximization from terminal wealth is one example for what we termed "other modules", we end up with an outlook on further research. In particular, we outline how we intend to include a limit order book into our analysis and how different levels of information affect price takers as well as the large trader.

The paper is structured as follows. In Section 2, we provide the definitions for the price process and the real wealth process for a simple large trader strategy. Besides, we introduce Assumptions I and II and give numerous examples and remarks to motivate and explain the definitions and assumptions. The main result is Proposition 2.8. It provides a link between Assumptions I and II. In Section 3, we extend the definition of the price process affected by the large trader and the definition of her real wealth process to general large trader strategies using a limit procedure. Furthermore, we highlight the different qualities of Assumptions I and II. While the leading edge of Assumption I is its flexibility, which is reflected in various examples, the strong suit of Assumption II is reflected in Theorem 3.3. The latter ensures the existence of price processes for general càglàd strategies. At the end, 
we provide a critical discussion regarding Assumptions I and II. Section 4 is dedicated to the analysis of no arbitrage conditions for the small trader given that the large trader uses general càglàd strategies. Here, the main results are Theorem 4.6 and Proposition 4.9. In Section 5, we turn to a case study on utility maximization from terminal wealth. In a plain setting, we illustrate various phenomena which may occur when prices are affected by a large trader. Section 6 provides the proofs, whereas Section 7 gives an outlook addressing further research questions. Finally, the Appendix provides an overview of strong nonlinear integrators and nonlinear stochastic integration.

2. Basic setting and examples for simple large trader strategies. Our financial market consists of a probability space $(\Omega, \mathcal{F}, \mathbb{P})$ equipped with a filtration $\mathbb{F}=\left(\mathcal{F}_{t}\right)_{t \in[0, T]}$ that satisfies the usual conditions. Besides, $\mathcal{F}_{0}$ is trivial apart from zero sets and $T$ denotes some finite time horizon. Furthermore, we want to stress the following points. Results from the literature, formulated for an infinite time horizon, are used by applying the corresponding result to the stopped process. Moreover, all relations between random variables are to be understood $\mathbb{P}$-a.s.

We consider two different types of investors: a small trader and a large trader. While we think of a small trader as a price taker, a large trader is an agent whose trades may influence the price of the asset she trades.

2.1. Primal family and the price process for simple large trader strategies. To allow for an impact of the large trader, the discounted price process is composed of three building blocks. The first one is the large trader's strategy.

DEFINITION 2.1. Let $\theta$ be a predictable process with representation

$$
\theta(t)=\theta_{-1} \mathbf{1}_{\{0\}}(t)+\sum_{i=0}^{n} \theta_{i} \mathbf{1}_{\left(\tau_{i}, \tau_{i+1}\right]}(t),
$$

where $0=\tau_{0} \leq \tau_{1} \leq \cdots \leq \tau_{n+1}=T$ is a finite sequence of $\mathbb{F}$-stopping times, $\theta_{-1} \in \mathbb{R}^{d}$ and $\theta_{i}$ is a bounded, $\mathcal{F}_{\tau_{i}}$-measurable, $\mathbb{R}^{d}$-valued random variable for all $i \in\{0, \ldots, n\}$.

1. We call $\theta$ an extended simple strategy if the random variables $\theta_{i}$ take only finitely many values.

2. An extended simple strategy is called simple strategy if the stopping times $\tau_{i}$ take only finitely many values.

3. A representation (3) is called minimal representation if the sequence of stopping times is deterministic and constitutes the smallest partition of $[0, T]$ that contains $0, T$, and all jump times of $\theta$ such that the jumps in these points occur with positive probability.

We denote the space of all $\mathbb{R}^{d}$-valued, extended simple strategies by $\mathbf{S}^{e}\left(\mathbb{R}^{d}\right)$. The space of all $\mathbb{R}^{d}$-valued simple strategies is denoted by $\mathbf{S}\left(\mathbb{R}^{d}\right)$. 
REMARK 2.2. 1. The closure of $\mathbf{S}\left(\mathbb{R}^{d}\right)$ and $\mathbf{S}^{e}\left(\mathbb{R}^{d}\right)$ w.r.t. the $u c p$-topology is the space of all $\mathbb{R}^{d}$-valued, càglàd and adapted processes; see [9], Proposition II.1.1. It is denoted by $\mathbb{L}\left(\mathbb{R}^{d}\right)$. Moreover, $b \mathbb{L}\left(\mathbb{R}^{d}\right) \subset \mathbb{L}\left(\mathbb{R}^{d}\right)$ denotes the subspace of all bounded càglàd processes.

2. While the spaces $\mathbf{S}\left(\mathbb{R}^{d}\right)$ and $\mathbf{S}^{e}\left(\mathbb{R}^{d}\right)$ are needed for technical reasons, the spaces $\mathbf{S}(\mathbb{R})$ and $\mathbb{L}(\mathbb{R})$ are considered as the spaces of all (simple) large trader trading strategies.

3. All $\theta \in \mathbf{S}(\mathbb{R})$ admit a unique minimal representation; see Lemma A.1.

4. Sometimes we need a càdlàg version of an element $\theta \in \mathbb{L}\left(\mathbb{R}^{d}\right)$. For all $\omega \in \Omega$, we denote it by $\theta^{+}=\left(\theta_{t}^{+}\right)_{t \in[0, T]}$, where $\theta_{T}^{+}:=\theta_{T}$ and $\theta_{t}^{+}(\omega):=\lim _{s \downarrow t} \theta_{s}(\omega)$ for $t \in[0, T)$.

The second and third building blocks that constitute the discounted price process affected by the large trader are the primal family $(S(\vartheta, \cdot))_{\vartheta \in \mathbb{R}^{d}}$ of $\mathbb{R}$-valued, $\mathbb{F}$ adapted, continuous semimartingales and an impact function

$$
\begin{aligned}
F: \mathbf{S}(\mathbb{R}) & \longrightarrow \mathbf{S}\left(\mathbb{R}^{d}\right), \\
\theta & \longmapsto F(\theta) .
\end{aligned}
$$

For a large trader strategy $\theta \in \mathbf{S}(\mathbb{R})$ and $F(\theta) \in \mathbf{S}\left(\mathbb{R}^{d}\right)$ with representation

$$
F_{t}(\theta)=F_{0}(\theta) \mathbf{1}_{\{0\}}(t)+\sum_{i=0}^{n} F_{\tau_{i}+}(\theta) \mathbf{1}_{\left(\tau_{i}, \tau_{i+1}\right]}(t),
$$

the discounted price process affected by the large trader is modelled by

$$
\begin{aligned}
P_{t}^{\theta} & :=\int_{0}^{t} S\left(F_{S}(\theta), d s\right) \\
& :=S\left(F_{0}(\theta), 0\right)+\sum_{i=0}^{n}\left(S\left(F_{\tau_{i}+}(\theta), \tau_{i+1} \wedge t\right)-S\left(F_{\tau_{i}+}(\theta), \tau_{i} \wedge t\right)\right) .
\end{aligned}
$$

Hereafter, we discuss the definition of $P^{\theta}$ as well as several assumptions on $P^{\theta}$ and the primal family $(S(\vartheta, \cdot))_{\vartheta \in \mathbb{R}^{d}}$ itself. We compare (6) and the assumptions to miscellaneous large trader models proposed in the literature. Among those are the models of Almgren and Chriss [2, 3], Bank and Baum [4], Gatheral [16], Jarrow [20] and the limit order book model of Alfonsi and Schied [1]. In order to give an economic interpretation of our definition of the discounted price process affected by the large trader, we consider only simple large trader strategies in this section. The definition of $P^{\theta}$ for $\theta \in \mathbb{L}(\mathbb{R})$ is postponed to Section 3 .

REMARK 2.3 (On the definition of $P^{\theta}$ ). The following ideas are behind the definition of $P^{\theta}$. First, the spatial parameters $\vartheta \in \mathbb{R}^{d}$ of the primal family $(S(\vartheta, \cdot))_{\vartheta \in \mathbb{R}^{d}}$ reflect all possible (constant) "impact levels" of the price process 
under consideration. Second, $F(\theta)$ is the actual impact of the large trader's strategy $\theta \in \mathbf{S}(\mathbb{R})$ on the price process. Hence, (6) constitutes a price process having an impact level that changes over time. More precisely, the stopping times $\left(\tau_{i}\right)_{i \leq n+1}$ in (5) are interpreted as those points in the future when the large trader's actions cause the changes $\left(F_{\tau_{i}+}(\theta)\right)_{i \leq n+1}$ of the impact level of the discounted price process.

REMARK 2.4 (On the impact function $F$ ). The task of the function $F$ in (6) is to incorporate different types of price impacts into the price process that are caused by the large trader's actions. Usually, one distinguishes between a permanent price impact and a temporary price impact. On the one hand, the permanent price impact affects the evolution of the price process even though the large trader does not trade. On the other hand, the temporary price impact usually vanishes whenever the large trader stops trading. These types of impact appear for example in the large trader model of Almgren and Chriss [2, 3]; see also Example 3.4 below. Apart from different types of price impacts, the function $F$ can also be used to model the wellknown resilience effect of prices after substantial trades of the large trader. An example of a model incorporating this effect is suggested by Gatheral; see [16] and Example 3.5 below. Finally, the impact function can be used in a more indirect way to include a volume impact limit order book into the model. An example is the model of Alfonsi and Schied [1]; see Example 7.1.

There are two aspects that we have to take into account. First of all, the price process affected by a large trader strategy should serve as a reasonable price process of a frictionless small trader model. Here, the class of semimartingales is the classical choice for modelling price processes in frictionless small trader markets. Second, the large trader's actions should not lead to arbitrage opportunities for the small trader. Hence, we work under the following standing assumption.

Assumption I. For all $\theta \in \mathbf{S}(\mathbb{R})$, the following hold:

$(P-I): P^{\theta}$ defined in (6) is a continuous semimartingale.

$(N A-I): \exists \mathbb{Q}^{\theta} \sim \mathbb{P}$ such that $P^{\theta}$ is a local $\mathbb{Q}^{\theta}$-martingale w.r.t. $\mathbb{F}$.

Remark 2.5 (On Assumption I). 1. The main idea of Assumption I is to consider only those price processes $P^{\theta}$ that do not contradict no-arbitrage assumptions for the small trader in a frictionless market. The probably most popular no-arbitrage assumption in a frictionless small trader market model is the no free lunch with vanishing risk condition. Due to the fundamental theorem of asset pricing ([12], Theorem 7.2 and Corollary 1.2), Assumption I is a natural consequence of the above considerations. Besides, this approach naturally embeds a large trader into the small trader setting.

2. The choice of the filtration $\mathbb{F}$ is for convenience. From an economic point of view, we think that it is more reasonable to consider different filtrations for the 
small and the large trader. In our opinion, the most natural choice for the filtration $\mathbb{F}^{S T}$, modelling the information of the small trader, is essentially the filtration generated by the discounted price process $P^{\theta}$. To us, the filtration $\mathbb{F}^{\mathrm{LT}}$ generated by $P^{\theta}$ and the large trader strategy $\theta$ would be the best choice for modelling the information of the large trader. Although a detailed investigation of phenomena appearing due to different levels of information is left for further research, we briefly discuss the main idea in Section 7.

3. For simple large trader strategies, $(N A-I)$ is essentially assumption four of Jarrow [20]. It claims that, given the large trader's information, there are no arbitrage opportunities for the small trader. The only difference is that the large trader's information is rather given by $\mathbb{F}^{\mathrm{LT}}$.

2.2. Regularity conditions on the primal family. On the one hand, the strength of the large trader impact on the price process strongly depends on the regularity and smoothness of the primal family $(S(\vartheta, \cdot))_{\vartheta \in \mathbb{R}^{d}}$ and the impact function $F$. The less regular $(S(\vartheta, \cdot))_{\vartheta \in \mathbb{R}^{d}}$ and $F$ are, the stronger is the impact of the large trader on the price process. On the other hand, a certain amount of regularity is needed to ensure that Assumption I holds. In this regard, the so-called strong nonlinear integrators, introduced in [9], constitute a quite general and yet tractable set of primal families satisfying (P-I). For a definition, we refer to the Appendix or [9]. As we will see below, the amount of regularity provided by strong nonlinear integrators allows us to work with a no-arbitrage assumption for the small trader that is weaker and even more tractable than (NA-I). It is formulated in Assumption II.

ASSUMPTION II. $\quad(P-I I):(S(\vartheta, \cdot))_{\vartheta \in \mathbb{R}^{d}}$ is a strong nonlinear integrator. Moreover, the function $F$ in (4) can be extended to a function (still denoted by) $F: \mathbb{L}(\mathbb{R}) \longrightarrow \mathbb{L}\left(\mathbb{R}^{d}\right)$ that is continuous w.r.t. the ucp-topology.

$(N A-I I)$ : For all $\vartheta \in \mathbb{R}^{d}$, there exists an equivalent local martingale measure $\mathbb{Q}^{\vartheta}$ for $S(\vartheta, \cdot)$ w.r.t. $\mathbb{F}$.

REMARK 2.6. Strong nonlinear integrators are tailor-made for our setting. On the one hand, they ensure by definition that $(P-I I)$ is sufficient for $(P-I)$. On the other hand, (6) is exactly the elementary nonlinear stochastic integral of $F(\theta) \in$ $\mathbf{S}\left(\mathbb{R}^{d}\right)$ w.r.t. the primal family $(S(\vartheta, \cdot))_{\vartheta \in \mathbb{R}^{d}}$.

REMARK 2.7 (Relation to the Bank and Baum model). If $d=1$ and $F=i d$, there are some similarities between our approach and the model proposed by Bank and Baum in [4].

1. Discounted price process: At the core of their model, Bank and Baum [4] also have a primal family $(S(\vartheta, \cdot))_{\vartheta \in \mathbb{R}}$ of continuous semimartingales in order to model the discounted price process affected by a large trader. Moreover, for constant large trader strategies $\vartheta \in \mathbb{R}$ our definition of the discounted price process 
coincides with the definition of Bank and Baum [4]. Hence, in the special case $F=i d$ we can interpret an element $S(\vartheta, \cdot)$ of the primal family as the discounted price process of a risky asset given the large trader holds a constant amount of $\vartheta$ shares in this asset. However, as soon as we consider nonconstant large trader strategies, the definitions of the price processes usually differ.

2. Regularity of the primal family: While we work under the regularity assumption $(P-I)$ or $(P-I I)$ on the primal family ([4], Assumption 1, of Bank and Baum), [4] asks for a different type of regularity. More precisely, it requires $(S(\vartheta, \cdot))_{\vartheta \in \mathbb{R}}$ to be a smooth family of semimartingales. This requirement is essentially a differentiability condition on $(S(\vartheta, \cdot))_{\vartheta \in \mathbb{R}}$ in the spatial parameter; see Kunita [25], Chapter III.

3. NA-condition for the small trader: The probably most important improvements of our approach are the no-arbitrage assumptions (NA-I) and (NA-II) concerning the small trader. While Bank and Baum [4] insist on the existence of a universal equivalent local martingale measure for all $S(\vartheta, \cdot)$, where $\vartheta \in \mathbb{R},(N A-I I)$ ensures that the small trader can not make riskless profits whenever the large trader does not trade.

Our first proposition highlights the link between (NA-I) and (NA-II) given that the primal family satisfies the regularity assumption (P-II).

Proposition 2.8. Suppose that $(S(\vartheta, \cdot))_{\vartheta \in \mathbb{R}^{d}}$ satisfies (P-II) and let $\tilde{\theta} \in$ $\mathbf{S}(\mathbb{R})$ be a simple strategy with representation (3). Under (NA-II), there exists an equivalent local martingale measure $\mathbb{Q}^{\tilde{\theta}}$ for the process $P^{\tilde{\theta}}$. In particular, (NA-II) is sufficient for (NA-I).

The following corollary is an immediate consequence of the definition of a strong nonlinear integrator and Proposition 2.8.

\section{COrollary 2.9. Assumption II implies Assumption I.}

The next theorem provides a sufficient condition for $(S(\vartheta, \cdot))_{\vartheta \in \mathbb{R}^{d}}$ to be a strong nonlinear integrator.

THEOREM 2.10. Let A be a nondecreasing, continuous, adapted process, $M$ be a continuous, local martingale with $\mathbb{E}\left[\sqrt{[M]_{T}}\right]<\infty$, and $\mu, \sigma: \mathbb{R}^{d} \times \mathbb{R} \longrightarrow \mathbb{R}$ jointly continuous functions. Let $(S(\vartheta, \cdot))_{\vartheta \in \mathbb{R}^{d}}$ be given by

$$
S(\vartheta, t)=S(\vartheta, 0)+\int_{0}^{t} \mu(\vartheta, S(\vartheta, u)) d A_{u}+\int_{0}^{t} \sigma(\vartheta, S(\vartheta, u)) d M_{u}
$$

and suppose that for almost all $\omega \in \Omega$ the mapping $S(\cdot, \cdot, \omega): \mathbb{R}^{d} \times[0, T] \longrightarrow \mathbb{R}$ is jointly continuous. Then $(S(\vartheta, \cdot))_{\vartheta \in \mathbb{R}^{d}}$ is a strong nonlinear integrator. 
2.3. Examples. We start with the probably most trivial example. Here, the large trader does not affect the price process at all.

EXAMPLE 2.11 (Classical small trader setting). If $S(\vartheta, \cdot)=S(0, \cdot)$ for all $\vartheta \in \mathbb{R}^{d}$, (6) coincides with the classical definition for a small trader market model. Indeed, the price process at time $t$ is $P_{t}^{0}$. In this case, the trading activities of the large trader have no impact on the evolution of the price process. Moreover, for all functions $F: \mathbf{S}(\mathbb{R}) \longrightarrow \mathbf{S}\left(\mathbb{R}^{d}\right),(N A-I)$ holds if and only if $P^{0}$ possesses an equivalent local martingale measure w.r.t. $\mathbb{F}$.

In the following two examples, $F=i d$ holds. This means that the price impact only depends on the large trader's position in the underlying asset.

EXAMPLE 2.12 (Stochastic differential equations). Let $d=1, F=i d$, and assume that processes $S(\vartheta, \cdot)$ are given as strong solutions of the SDEs

$$
d S(\vartheta, t)=b^{\vartheta}(S(\vartheta, t)) d t+\sigma(S(\vartheta, t)) d W_{t} .
$$

Here, $W$ is a Brownian motion and the function $b: \mathbb{R} \times \mathbb{R} \longrightarrow \mathbb{R}$, where $(\vartheta, x) \longmapsto$ $b^{\vartheta}(x)$, is assumed to be continuous and nondecreasing in the first argument and Lipschitz continuous in the second argument. Furthermore, we assume that $\sigma$ is a function that is bounded from below by some $\varepsilon>0$ and satisfies $|\sigma(x)-\sigma(y)|^{2} \leq$ $\rho|x-y|$ for some $\rho>0$. Besides, let $S(\vartheta, 0) \leq S\left(\vartheta^{\prime}, 0\right)$ whenever $\vartheta \leq \vartheta^{\prime}$. Note that the comparison theorem [29], (3.7) Theorem, for SDEs ensures that the family $(S(\vartheta, \cdot))_{\vartheta \in \mathbb{R}}$ satisfies the following.

Condition $(O): \vartheta \leq \vartheta^{\prime}$ implies $S(\vartheta, \cdot) \leq S\left(\vartheta^{\prime}, \cdot\right)$.

This condition has been introduced by Bank and Baum [4]. Due to Theorem 2.10 and Lemma A.10, we can conclude that $(S(\vartheta, \cdot))_{\vartheta \in \mathbb{R}}$ satisfies $(P-I I)$. Besides if, for example, $\left(b^{\vartheta}\right)_{\vartheta \in \mathbb{R}}$ is a family of bounded functions, Girsanov's theorem and Novikov's criterion guarantee that assumption (NA-II) holds. However, there is in general no universal equivalent local martingale measure for all $S(\vartheta, \cdot)$. Thus, the analysis of Bank and Baum [4] does not apply to this situation. Finally, we want to emphasize the following. Condition $(O)$ has been introduced by Bank and Baum [4] to exclude arbitrage opportunities for the large trader in their model. We do not need Condition $(O)$ for any particular reason. It is just an additional feature of this primal family.

EXAMPLE 2.13 (Reaction-diffusion setting). This example is similar to the model of Frey [15]. Let $\psi(t, x, \vartheta)$ be a $\mathcal{C}^{1,2,1}$-function, $F=i d$, and $d=1$. Define $(S(\vartheta, \cdot))_{\vartheta \in \mathbb{R}}$ via $S(\vartheta, t)=\psi\left(t, W_{t}, \vartheta\right)$, where the Brownian motion $W$ models some fundamental state variable. Due to Itô's formula, the dynamics of the primal price processes have the form

$$
d \psi_{t}=\left(\frac{\partial}{\partial t}+\frac{1}{2} \frac{\partial^{2}}{\partial x^{2}}\right) \psi_{t} d t+\frac{\partial}{\partial x} \psi_{t} d W_{t}
$$


We assume that $\partial \psi / \partial x$ is strictly positive. Note that $\partial \psi / \partial \vartheta \geq 0$ implies Condition $(O)$. An explicit example for a reaction function is given by $\psi\left(t, W_{t}, \vartheta\right)=$ $\exp \left(\sigma W_{t}+\kappa \vartheta t\right)$, where $\sigma, \kappa>0$. Its dynamics are given by

$$
d \psi_{t}=\psi_{t}\left(\left(\kappa \vartheta+\frac{1}{2} \sigma^{2}\right) d t+\sigma d W_{t}\right) .
$$

Moreover, $(P-I I)$ holds thanks to Theorem 2.10 and Lemma A.10. Again, Girsanov's theorem and Novikov's criterion ensure that (NA-II) holds in this particular example. But there does not exist a universal local martingale measure for all $S(\vartheta, \cdot)$. Thus, the analysis of Bank and Baum [4] does not apply to the reactiondiffusion setting.

At this point, we want to call attention to the fact that not only the primal families, but also the price processes in the last three examples are strong nonlinear integrators. This is essentially due to the fact that the function $F$ is the identity. In the next example, we call it the additive compound impact model, only the primal family is a strong nonlinear integrator. Although (P-I) holds, the model does not necessarily satisfy (P-II).

EXAMPLE 2.14 (Additive compound impact model). The underlying price process in the additive compound impact model is given by the sum of an unperturbed price $\tilde{P}$ and a price impact $I^{\theta}$ that depends on the large trader's actions. More precisely, for $\theta \in \mathbf{S}(\mathbb{R})$ the price process is given by

$$
P^{\theta}=\tilde{P}+I^{\theta}
$$

where $\tilde{P}$ is a continuous semimartingale and $P^{\theta}$ satisfies Assumption I. A typical example is given by $I^{\vartheta}=t \sum_{i \leq d} \vartheta_{i}$ for $\vartheta \in \mathbb{R}^{d}$ and

$$
\tilde{P}=\tilde{P}_{0}+\sigma W
$$

where $\sigma>0$ and $W$ is a Brownian motion. Hence, for $F: \mathbf{S}(\mathbb{R}) \longrightarrow \mathbf{S}\left(\mathbb{R}^{d}\right)$, where $F(\theta)=\left(F^{1}(\theta), \ldots, F^{d}(\theta)\right)$, and a continuous function $I: \mathbb{R}^{d} \longrightarrow \mathbb{R}$ with $\vartheta \longmapsto$ $I(\vartheta, 0)$, we get

$$
I_{t}^{\theta}=I\left(F_{0}(\theta), 0\right)+\sum_{i=1}^{d} \int_{0}^{t} F_{u}^{i}(\theta) d u
$$

The primal family in the last example serves as the primal family for the large trader models of Almgren and Chriss [2, 3], Gatheral [16] and Alfonsi and Schied [1]. We discuss them extensively in the next section.

2.4. The large trader real wealth process for simple strategies. To incorporate liquidity risk into their large trader model, Bank and Baum [4] use a special 
definition of the bank account as well as an idealized definition of the real wealth process, the asymptotic liquidation proceed process. In Çetin et al. [10], the authors incorporate liquidity risk into their model by using a new definition of selffinancing strategies. Using particular integration by parts formulas, both conclude that the real wealth process $V(\theta)$ of the large trader is of the form

$$
V(\theta)=V_{0}+G(\theta)-C(\theta) .
$$

While $G(\theta)$ represents the gains and losses due to trading, the nonnegative process $C(\theta)$ is interpreted as costs due to liquidity risk. Similar to [4] and [10], we only consider self-financing large trader strategies. In order to define self-financing strategies and to incorporate liquidity risk into our model, we choose an approach being a mixture of [4] and [10]. For simple large trader strategies, we define the cost function, linked to liquidity risk, as

$$
\begin{aligned}
C: \mathbf{S}(\mathbb{R}) & \longrightarrow \mathcal{V}_{\geq 0}, \\
\theta & \longmapsto C_{0}^{\theta}+C(\theta) .
\end{aligned}
$$

Here, $C_{0}^{\theta} \in \mathbb{R}, C_{0}(\theta)=0$ for all $\theta \in \mathbf{S}(\mathbb{R})$, and $\mathcal{V}_{\geq 0}$ denotes the set of all adapted, nonnegative càdlàg processes of finite variation. A strategy $\left(\beta^{\theta}, \theta\right)$, where $\theta \in \mathbf{S}(\mathbb{R})$ and $\beta^{\theta}$ is an optional process, is called self-financing if

$$
\beta_{t}^{\theta}=\beta_{0}^{\theta}-\int_{0}^{t} P_{u}^{\theta} d \theta_{u}^{+}-C_{0}^{\theta}-C_{t}(\theta)
$$

where $\theta^{+}$is defined in Remark 2.2. As we work with discounted price processes, $\beta^{\theta}$ can be interpreted as the money market account. Hence, the product rule leads to the following reformulation of the self-financing condition:

$$
\beta_{t}^{\theta}+\theta_{t}^{+} P_{t}^{\theta}=\beta_{0}^{\theta}+\theta_{0} P_{0}^{\theta}-C_{0}^{\theta}+\int_{0}^{t} \theta_{u} d P_{u}^{\theta}-C_{t}(\theta)
$$

Note that for $C \equiv 0$ and $P^{\theta}=P^{0}$ for all $\theta \in \mathbf{S}(\mathbb{R})$, the last equation is the classical definition of a self-financing strategy in a frictionless small trader market model. These considerations lead us to the following definition of the large trader real wealth process $V(\theta)$ for $\theta \in \mathbf{S}(\mathbb{R})$. If we set $V_{0}^{\theta}:=\beta_{0}^{\theta}+\theta_{0} P_{0}^{\theta}-C_{0}^{\theta}$, it is given by

$$
V_{t}(\theta)=V_{0}^{\theta}+\int_{0}^{t} \theta_{u} d P_{u}^{\theta}-C_{t}(\theta)
$$

REMARK 2.15. Suppose that, as in Example 2.14, the price process $P^{\theta}$ can be decomposed into the sum of two semimartingales $\tilde{P}$ and $I^{\theta}$. As a result, the accumulated gains and losses $G(\theta)=\int \theta d P^{\theta}$ are given by

$$
G(\theta)=\int \theta d \tilde{P}+\int \theta d I^{\theta}
$$


Clearly, if $P^{\theta}$ can be decomposed as above, the real wealth process of a selffinancing simple strategy $\theta$ is given by

$$
V(\theta)=V_{0}^{\theta}+\int \theta d \tilde{P}+\int \theta d I^{\theta}-C(\theta) .
$$

While $\int \theta d \tilde{P}$ accounts for the accumulated gains and losses from stock price fluctuations caused by exogenous random shocks, the term $\int \theta d I^{\theta}-C(\theta)$ represents profits and losses due to liquidity risks and the price impact of the large trader.

We end this section with some examples for cost functions.

EXAMPLE 2.16 (Cost functions). Let $\theta \in \mathbf{S}(\mathbb{R})$ and $\epsilon>0$.

1. Zero cost function: The most simple cost function is the constant zero function, that is, $C(\theta) \equiv 0$. However, the term $\int \theta d I^{\theta}$ in (10), representing the price impact of the large trader, might still lead to costs due to liquidity risks; see Example 3.4 below.

2. Maximal change cost function: This cost function is defined as follows:

$$
\text { C. }(\theta)=\max \left\{\left|\theta_{0}^{+}-\theta_{0}\right|, \sup _{s \leq .}\left|\theta_{s}^{+}-\theta_{0}^{+}\right|\right\} .
$$

Graphically, $\theta$ is the costlier the more it deviates from its initial value.

3. Proportional (variation) costs: Denote by $\operatorname{Var}^{\theta}$ the total variation process of $\theta^{+}$. Similar to the terms that are used to model proportional transaction costs, we define

$$
C(\theta)=\epsilon\left(\left|\theta_{0}^{+}-\theta_{0}\right|\left|P_{0}^{\theta}\right|+\int\left|P^{\theta}\right| d \operatorname{Var}^{\theta}\right) .
$$

The cost function ties liquidity risk to a fraction of the trading volume.

4. Proportional (quadratic variation) costs: Suppose that $d=1, F=i d$, and that Condition $(O)$ holds. Moreover, we assume that the primal family $(S(\vartheta, \cdot))_{\vartheta \in \mathbb{R}}$ is continuously differentiable in the spatial parameter and denote this derivative by $\left(S^{\prime}(\vartheta, \cdot)\right)_{\vartheta \in \mathbb{R}}$. Since $F=i d$, we have $\left(P^{\vartheta}\right)_{\vartheta \in \mathbb{R}}=(S(\vartheta, \cdot))_{\vartheta \in \mathbb{R}}$ as well as $\left(\frac{\partial}{\partial \vartheta} P^{\vartheta}\right)_{\vartheta \in \mathbb{R}}=\left(S^{\prime}(\vartheta, \cdot)\right)_{\vartheta \in \mathbb{R}}$. Define

$$
\begin{aligned}
\frac{1}{\epsilon} C .(\theta) & :=\sum_{0 \leq s \leq .} \int_{\theta_{s-}^{+}}^{\theta_{s}^{+}}\left(S\left(\theta_{s}^{+}, s\right)-S(x, s)\right) d x \\
& =\frac{1}{2} \int_{0}^{\cdot} S^{\prime}\left(\theta_{s-}^{+}, s\right) d\left[\theta^{+}\right]_{s}^{c}+\sum_{0 \leq s \leq .} \int_{\theta_{s-}^{+}}^{\theta_{s}^{+}}\left(S\left(\theta_{s}^{+}, s\right)-S(x, s)\right) d x
\end{aligned}
$$

and note that the second equality follows by applying the integration by parts formula to

$$
\int_{\theta_{s-}^{+}}^{\theta_{s}^{+}}\left(S\left(\theta_{s}^{+}, s\right)-S(x, s)\right) d x=\int_{\theta_{s-}^{+}}^{\theta_{s}^{+}} \frac{\left(S\left(\theta_{s}^{+}, s\right)-S(x, s)\right)}{\theta_{s}^{+}-x}\left(\theta_{s}^{+}-x\right) d x
$$


and rearranging the resulting terms. Note that this is exactly the cost function (related to liquidity risk) derived by Bank and Baum [4] using the Itô-Wentzell formula. We would like to point out that Çetin et al. [10] derive a similar formula reflecting the costs due to liquidity risks. Indeed, in both models the costs due to liquidity risk strongly depend on the quadratic variation of the large trader strategy.

3. Price process and real wealth process for dynamic large trader strategies. In this section, we provide definitions of the price process $P^{\theta}$ and the large trader real wealth process for a general strategy $\theta \in \mathbb{L}(\mathbb{R})$. Our main goal is to ensure that these definitions translate the ideas of Assumption I to general strategies. Furthermore, we provide examples highlighting that (P-II) does not hold in the large trader models of Almgren and Chriss [2, 3] and Gatheral [16]. Besides, we provide a critical discussion of Assumptions I and II and the definitions of price process and real wealth process for general large trader strategies.

3.1. The price process for dynamic strategies. We start with the definition of the discounted price process $P^{\theta}$ for $\theta \in \mathbb{L}(\mathbb{R})$.

Definition 3.1. Let $(S(\vartheta, \cdot))_{\vartheta \in \mathbb{R}^{d}}$ satisfy $(P-I)$ and $\theta \in \mathbb{L}(\mathbb{R})$. If there exists a sequence $\left(\theta^{n}\right)_{n \geq 1} \subset \mathbf{S}(\mathbb{R})$ such that:

1. $\theta^{n} \longrightarrow \theta$ in the ucp-topology,

2. $\left(P^{\theta^{n}}\right)_{n \geq 1}$ is a Cauchy sequence in the semimartingale topology,

then the discounted price process $P^{\theta}$ affected by the large trader strategy $\theta \in \mathbb{L}(\mathbb{R})$ is the limit of $\left(P^{\theta^{n}}\right)_{n \geq 1}$ in the semimartingale topology, that is,

$$
P^{\theta^{n}} \underset{\mathcal{S M}}{\longrightarrow} P^{\theta}
$$

The next lemma is a tool to verify whether or not $P^{\theta}$ exists for $\theta \in \mathbb{L}(\mathbb{R})$.

LEMMA 3.2. Let $\theta \in \mathbb{L}(\mathbb{R}),\left(\theta^{n}\right)_{n \geq 1} \subset \mathbb{L}(\mathbb{R})$, and suppose that $P^{\theta^{n}}$ exists for all $n \geq 1$. If $\theta^{n} \longrightarrow \theta$ in the ucp-topology and $\left(P^{\theta^{n}}\right)_{n \geq 1}$ is a Cauchy sequence in the semimartingale topology, then $P^{\theta}$ exists in the sense of Definition 3.1 and $P^{\theta^{n}} \rightarrow P^{\theta}$ in the semimartingale topology.

We already argued that (P-II) might be too restrictive in some cases. Nevertheless, it ensures the existence of price processes affected by the large trader for arbitrary strategies. The next statement is an immediate consequence of the definition of strong nonlinear integrators and Theorem A.16.

THEOREM 3.3. If $(S(\vartheta, \cdot))_{\vartheta \in \mathbb{R}^{d}}$ satisfies $(\mathrm{P}-\mathrm{II})$, then for all $\theta \in \mathbb{L}(\mathbb{R})$ there exists $\left(\theta^{n}\right)_{n \geq 1} \subset \mathbf{S}(\mathbb{R})$ such that the following properties hold: 
1. $\theta^{n} \longrightarrow \theta$ in the ucp-topology.

2. $\left(P^{\theta^{n}}\right)_{n \geq 1}$ is a Cauchy sequence in the semimartingale topology.

Thus, the discounted price process $P^{\theta}$ affected by the large trader strategy $\theta$ exists. Finally, the definition does not depend on the approximating sequence.

Due to Theorem 3.3, $P^{\theta}$ in Examples 2.11, 2.12 and 2.13 exists for all $\theta \in \mathbb{L}(\mathbb{R})$. However, the next two examples, the large trader models of Almgren and Chriss $[2,3]$ and Gatheral [16], are typical examples in which the impact function $F$ is not smooth enough to ensure that (P-II) holds. Nevertheless, (P-I) holds and for sufficiently regular large trader strategies $\theta \in \mathbb{L}(\mathbb{R})$ the price process $P^{\theta}$ exists in the sense of Definition 3.1.

EXAMPLE 3.4 (Almgren-Chriss model). The Almgren-Chriss model $[2,3]$ can be reached as the limit of a special case of the additive compound impact model; see Example 2.14. Let $g, h \in C(\mathbb{R}, \mathbb{R})$ be nondecreasing, continuous functions, where $h \in C^{1}(\mathbb{R}, \mathbb{R})$ and $g(0)=h(0)=0$, and set

$$
\begin{aligned}
\mathcal{X}:= & \{\theta \in b \mathbb{L}(\mathbb{R}) \mid \theta \text { has twice cont. diff. paths on }(0, T], \\
& \left.\lim _{u \longrightarrow 0} \dot{\theta}_{u} \text { exists and equals } \theta_{0}\right\} .
\end{aligned}
$$

For $\theta \in \mathcal{X}$, the price process is given by

$$
P^{\theta}=\tilde{P}+I^{\theta},
$$

where $\tilde{P}$ is defined as in (7) and the price impact $I^{\theta}$ is given by

$$
I^{\theta}=\int g(\dot{\theta}) d u+h(\dot{\theta}) .
$$

Usually, the term $\int g(\dot{\theta}) d u$ is referred to as permanent price impact while $h(\dot{\theta})$ is called a temporary price impact.

For $\theta \in \mathbf{S}(\mathbb{R})$, we define $F: \mathbf{S}(\mathbb{R}) \longrightarrow \mathbf{S}\left(\mathbb{R}^{2}\right)$ in Example 2.14 as follows: while $F^{1}$ maps the minimal representation,

$$
\theta(t)=\theta_{-1} \mathbf{1}_{\{0\}}+\sum_{i=0}^{n} \theta_{t_{i}+} \mathbf{1}_{\left(t_{i}, t_{i+1}\right]}(t)
$$

of $\theta \in \mathbf{S}(\mathbb{R})$ to

$$
F^{1}(\theta)=h\left(\theta_{-1}\right) \mathbf{1}_{\{0\}}+\sum_{i=0}^{n} g\left(\frac{\theta_{t_{i}+}-\theta_{t_{i-1}}+}{t_{i}-t_{i-1}}\right) \mathbf{1}_{\left(t_{i}, t_{i+1}\right]}(t),
$$

$F^{2}$ maps the minimal representation of $\theta \in \mathbf{S}(\mathbb{R})$ to

$$
F^{2}(\theta)= \begin{cases}\sum_{i=4}^{n} \frac{\theta_{t_{i}+}-2 \theta_{t_{i-2}+}+\theta_{t_{i-4}+}}{\left(\frac{t_{i}-t_{i-4}}{2}\right)^{2}} h^{\prime}\left(\frac{\theta_{t_{i}+}-\theta_{t_{i-1}+}}{t_{i}-t_{i-1}}\right) \mathbf{1}_{\left(t_{i}, t_{i+1}\right]}(t) & \text { if } n \geq 4, \\ 0 & \text { else. }\end{cases}
$$


Finally, we choose $I: \mathbb{R}^{2} \longrightarrow \mathbb{R}$, where $\left(\vartheta_{1}, \vartheta_{2}\right) \longmapsto \vartheta_{1}$. For $\theta \in \mathcal{X}$, we define the approximating sequence $\left(\theta^{n}\right)_{n \geq 1}$ via

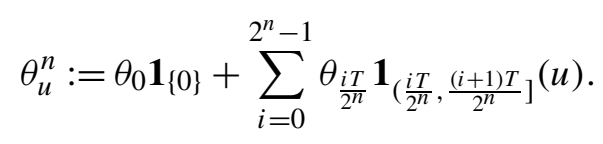

Due to Proposition A.7, it is straightforward to check that $\left(P^{\theta^{n}}\right)_{n \geq 1}$ exists. As $\theta^{n} \longrightarrow \theta$ in the $u c p$-topology, Taylor's theorem and the dominated convergence theorem allow us to conclude that for all $\omega \in \Omega$ the sequence $\left(I^{\theta^{n}}(\omega)\right)_{n \geq 1}$ converges in $L^{1}([0, T], L e b)$ to $I^{\theta}(\omega)$ as defined in (11). Hence, Proposition A.7 guarantees that $P^{\theta^{n}} \rightarrow P^{\theta}$ in the semimartingale topology. Due to Lemma 3.2, $P^{\theta}$ is a discounted price process in the sense of Definition 3.1 for all $\theta \in \mathcal{X}$. Finally, if $\theta$ has bounded first and second derivatives, Girsanov's theorem and Novikov's criterion imply that there exists an equivalent local martingale measure for $P^{\theta}$.

EXAMPLE 3.5 (Gatheral model with exponential decay). Similar to the Almgren-Chriss model [2,3], we can reach the model of Gatheral [16] as the limit of a special case of the additive compound impact model. Let $h: \mathbb{R} \longrightarrow \mathbb{R}$ be a nondecreasing, continuous function with $h(0)=0, \eta>0$, and define

$$
\mathcal{X}:=\{\theta \in b \mathbb{L}(\mathbb{R}) \mid \theta \text { has continuously differentiable paths }\} .
$$

For $\theta \in \mathcal{X}$, the price process is given by

$$
P^{\theta}=\tilde{P}+I^{\theta},
$$

where $\tilde{P}$ is defined as in (7) and the price impact $I^{\theta}$ is given by

$$
I_{t}^{\theta}=\int_{0}^{t} h\left(\dot{\theta}_{u}\right) e^{-\eta(t-u)} d u .
$$

This price impact is neither temporary nor permanent. It rather reflects the resilience effect of prices after a sudden and major trade of the large trader.

Due to the product rule, we can rewrite $I_{t}^{\theta}=e^{-\eta t} X_{t}^{\theta}$ as

$$
I_{t}^{\theta}=\int_{0}^{t} \eta\left(h\left(\dot{\theta}_{u}\right)-X_{u}^{\theta} e^{-\eta u}\right) d u,
$$

where $X_{u}^{\theta}:=\int_{0}^{u} h\left(\dot{\theta}_{v}\right) e^{\eta v} d v$. Denote by (12) the minimal representation of $\theta \in \mathbf{S}(\mathbb{R})$, let

$$
\tilde{\theta}=\sum_{i=0}^{n} h\left(\frac{\theta_{t_{i}+}-\theta_{t_{i-1}+}}{t_{i}-t_{i-1}}\right) \mathbf{1}_{\left.t_{i}, t_{i+1}\right]}(t),
$$

define $Y_{u}^{\tilde{\theta}}:=\int_{0}^{u} \tilde{\theta}_{v} e^{\eta v} d v$, and let $F: \mathbf{S}(\mathbb{R}) \longrightarrow \mathbf{S}(\mathbb{R}) \operatorname{map} \theta \in \mathbf{S}(\mathbb{R})$ to

$$
\eta \tilde{\theta}_{u}-\eta \sum_{i=0}^{n} Y_{t_{i}}^{\tilde{\theta}} e^{-\eta t_{i}} \mathbf{1}_{\left(t_{i}, t_{i+1}\right]}(u) .
$$


If we choose the approximating sequence $\left(\theta^{n}\right)_{n \geq 1}$ for $\theta \in \mathcal{X}$ as in (13), similar arguments as in Example 3.4 ensure that $\left(P^{\theta^{n}}\right)_{n \geq 1}$ and $P^{\theta}$ exist, $P^{\theta^{n}} \rightarrow P^{\theta}$ in the semimartingale topology, and (14) holds. Finally, if $\theta$ has bounded first derivatives, Girsanov's theorem and Novikov's criterion ensure that there exists an equivalent local martingale measure for $P^{\theta}$.

REMARK 3.6. 1. To ease the representation of the above examples, we used an approximating sequence (13) that does not constitute of simple strategies in the sense of Definition 2.1. This is due to the fact that $\theta_{\frac{i T}{2^{n}}}$ does not necessarily take finitely many values. However, using Lemma 3.2 and the fact that bounded random variables can be approximated by bounded random variables having only finitely many values, this gap can easily be fixed.

2. The volume impact model of Alfonsi and Schied [1] is yet another example that highlights the difference between $(P-I)$ and $(P-I I)$; see Example 7.1 below.

3.2. The large trader real wealth process for dynamic strategies and a critical discussion of Assumptions I and II. For general strategies, the cost function as well as the self-financing condition are defined by using a limit procedure.

Definition 3.7. Suppose that Assumption I holds and let $C: \mathbf{S}(\mathbb{R}) \longrightarrow \mathcal{V}_{\geq 0}$. We say that $\theta \in \mathbb{L}(\mathbb{R})$ is self-financing if there exists a sequence $\left(\theta^{n}\right)_{n \geq 1} \subset \mathbf{S}(\mathbb{R})$ of self-financing simple strategies such that:

1. $\theta^{n}$ converges in the $u c p$-topology to $\theta \in \mathbb{L}(\mathbb{R})$.

2. $P^{\theta}$ exists in the sense of Definition 3.1 w.r.t. this particular sequence.

3. The sequence $\left(C\left(\theta^{n}\right)\right)_{n \geq 1}$ converges in the semimartingale topology. Its limit, denoted by $C(\theta)$, is interpreted as the liquidity risk related to $\theta$.

In particular, the real wealth process of $\theta$ is defined as in (8). Moreover, we call the self-financing large trader strategy $\theta$ tame if $C(\theta) \equiv 0$. Finally, for a self-financing strategy $\theta \in \mathbb{L}(\mathbb{R})$, we call $\left((S(\vartheta, \cdot))_{\vartheta \in \mathbb{R}^{d}}, F, C, \theta\right)$ a minimal large trader market model if there exists an equivalent local martingale measure for $P^{\theta}$ w.r.t. the filtration $\mathbb{F}$.

REMARK 3.8. The phrase "minimal large trader market model" indicates that the large trader model satisfies the minimal requirements needed to:

1. define the discounted price process,

2. exclude arbitrage opportunities for the small trader, and

3. ensure that we can define the real wealth process of the large trader.

Until the end of this section, we discuss our definition of the price process affected by a large trader strategy in the context of Assumption I/(P-I), Assumption II/(P-II), and the large trader real wealth process. 
Clearly, Assumption I and Assumption II, in combination with Theorem 3.3, are extreme points of a spectrum of assumptions that one might formulate in order to ensure the existence of price processes for a large class of large trader strategies. In our opinion, Assumption I is the minimal assumption required by reality. This is due to the fact that in practice one can only trade using finite linear combinations of buy and hold strategies. From a mathematical point of view, it is desirable to seek for assumptions that also ensure the existence of the price process for a large class of nonsimple dynamic strategies. Assumption II/(P-II) is one example that allows to define the price processes for all adapted processes having càglàd paths. This class of strategies is the classical class of strategies/integrands suggested by stochastic integration theory. Hence, these assumptions are extreme points of a spectrum of assumptions focusing on different aspects, reality and theory. Unfortunately, Assumption II/(P-II) is too strong in order to include the classical large trader models of Example 3.4 and Example 3.5 that are frequently used in practice. Thus, one could ask for an assumption that provides a better trade off between theory and reality. We believe that there are two ways to come up with such an assumption. The first one is to impose more mathematical assumptions, for example, on the primal family or the impact function that allow us to conclude that the price process exists for a sufficiently large class of nonsimple, dynamic strategies. However, this way the "theory" (in terms of the postulated assumptions) chooses the strategies. The second way is to postulate a minimal assumption (Assumption I) that is as close to reality as possible. This "close to reality model" chooses the set of strategies. The next remarks provide more insights into these approaches.

REMARK 3.9 (On: "The model chooses the strategies"). How does the model choose the strategies? We have already argued in the Introduction, that the most important question is the following: "What is the large trader's intention to trade?" In a mathematical context, these questions are usually related to the large trader's real wealth process. A typical example is utility maximization from terminal wealth. While under Assumption I, we can define the real wealth process as well as the price process affected by simple large trader strategies, for general strategies these processes may not exist. But if the large trader's real wealth process does not exist, we have no tool to decide whether or not this strategy is important from an economic point of view. Yet, for special choices of the primal family and the cost function one can presume that economic considerations limit the set of reasonable large trader strategies significantly, that is, the model chooses its own strategies. Let us consider an example.

Consider the cost function in Example 2.16 4. It is clear that all strategies having continuous paths of finite variation admit zero liquidity costs, that is, they are tame. Now suppose that $P^{\theta}$ exists for all these strategies. Moreover, suppose that for all $\epsilon>0$ and all $\theta \in \mathbb{L}(\mathbb{R})$ such that $P^{\theta}$ exists, there exists $\theta^{\epsilon} \in \mathbb{L}(\mathbb{R})$ having continuous paths of finite variation and

$$
\mathbb{P}\left(\sup _{s \leq T}\left|\int_{0}^{s} \theta_{u} d P_{u}^{\theta}-\int_{0}^{s} \theta_{u}^{\epsilon} d P_{u}^{\theta^{\epsilon}}\right|<\epsilon\right)=1 .
$$


It is then clear that it suffices to consider only strategies having continuous paths of finite variation. Nevertheless, it is the model having chosen this class of strategies. However, it is not clear whether or not this holds if we just use another cost function, for example, the cost function in Example 2.16 2. Finally, we would like to mention that such approximation results (even for nonlinear integrators) exist under certain technical conditions; see, for example, [4], Theorem 4.4. Particularly, this line of arguments is used in [4] and [10] to reduce their analysis to strategies of finite variation having continuous paths.

REMARK 3.10 (On: "The theory chooses the strategies"). Clearly, the approach described in Remark 3.9 has one disadvantage. It does not provide an easyto-handle recipe that ensures the existence of price processes for a large class of large trader strategies. At this point, additional assumptions, motivated by mathematical theory, come into play. One example is Assumption II/(P-II). Although this type of assumptions focuses on technical necessities, these assumptions also reflect a fair bit of specific features of the object that an author wants to model. For example, Assumption II/(P-II) enables us to use the theory of nonlinear stochastic integration in the sense of Carmona and Nualart [9]. In particular, it is "tailormade" to extend the analysis of the no-arbitrage property for the small trader to price processes $P^{\theta}$ affected by general large trader strategies; see Section 4 below. In our opinion, another big advantage of Assumption II/(P-II) is the fact that it does not fix explicit mechanics of the impact a large trader strategy has on the price process. Of course, if one "knows how" the large trader influences the price, one can propose more specific and easy to handle assumptions. Suppose, for example, that one knows that the speed of changes in the large trader's strategy is responsible for the price impact. Then it seems to be plausible to use the first derivative of a trading strategy in order to model this impact. In particular, it is obvious that one chooses strategies having continuously differentiable paths to come up with an Almgren-Chriss-type model.

We end this section with a lemma that provides an example for a sufficient assumption for the additive compound impact model introduced in Example 2.14. As the structure of the primal family is known, we are able to formulate tailor-made assumptions that ensure the existence of the price process for a large set of nonsimple large trader strategies. Its proof is an application of Lebesgue's dominated convergence theorem and Proposition A.7.

LEMMA 3.11. Let $\theta \in \mathbb{L}(\mathbb{R})$ and consider the primal family of the additive compound impact model, where $\tilde{P}$ is an arbitrary continuous semimartingale and $\left(I^{\vartheta}\right)_{\vartheta \in \mathbb{R}^{d}}$ is defined as in Example 2.14. Then $P^{\theta}$ exists if there exists $\left(\theta^{n}\right)_{n \geq 1} \subset$ $\mathbf{S}(\mathbb{R})$ such that the following holds:

1. $\theta^{n} \longrightarrow \theta$ in the ucp-topology.

2. $\left(F\left(\theta^{n}\right)\right)_{n \geq 1}$ is a Cauchy sequence in the ucp-topology. 
3. There exists a nonnegative process $\bar{\theta}$ such that $\int_{0}^{T} \bar{\theta}_{u} d u<\infty \mathbb{P}$-a.s. and $\mathbb{P}\left(\left\|F_{t}\left(\theta^{n}\right)\right\|_{\mathbb{R}^{d}} \leq \bar{\theta}_{t} \forall t \leq T\right)=1$ holds for all $n \geq 1$.

REMARK 3.12. Whenever the impact function $F$ is given explicitly, it is likely that the limit can be identified; compare, for example, Examples 3.4 and 3.5.

4. No arbitrage condition for dynamic strategies under Assumption II. In this section, we assume that Assumption II holds. Thanks to Proposition 2.8, the regularity of the primal family also provides a regularity of the no-arbitrage assumptions for the small trader, that is, (NA-II) is sufficient for (NA-I). Besides, Theorem 3.3 ensures that $P^{\theta}$ exists for all strategies $\theta \in \mathbb{L}(\mathbb{R})$. The goal of this section is to provide sufficient conditions that enable us to extend the result of Proposition 2.8 to general $\theta \in \mathbb{L}(\mathbb{R})$.

REMARK 4.1. If $F=i d$, Proposition A.14 ensures that the statement of Proposition 2.8 also holds for $\theta \in \mathbf{S}^{e}(\mathbb{R})$.

Simply put, we are looking for sufficient conditions ensuring that the large trader's actions, that is, her strategy $\theta \in \mathbb{L}(\mathbb{R})$, do not lead to arbitrage opportunities for the small trader. Our main tool is the following version of the fundamental theorem of asset pricing. It follows directly from [12] and [18], Theorem 3.4. Recall that a continuous semimartingale $S$ satisfies the structure condition (SC) if its canonical decomposition is given by $S=S_{0}+M+\int \lambda d[M]$, where $M$ is a continuous local martingale starting in zero and $\int_{0}^{T} \lambda_{u}^{2} d[M]_{u}<\infty$ holds $\mathbb{P}$-a.s.

THEOREM 4.2. Let $S=M+\int \lambda d[M]$ be a continuous semimartingale:

1. S satisfies (SC) if and only if $\mathcal{E}\left(-\int \lambda d M\right)$ is a strictly positive local martingale density for $S$, that is, $\mathcal{E}\left(-\int \lambda d M\right) S$ is a local $\mathbb{P}$-martingale.

2. There exists an equivalent local martingale measure for $S$ if and only if $S$ satisfies (SC) and the classical (NA)-condition, that is, for all $S$-integrable processes $H$ that satisfy $\mathbb{P}\left(\int_{0}^{t} H_{u} d S_{u} \geq-1 ; \forall t \leq T\right)=1$, the following implication holds:

$$
\mathbb{P}\left(\int_{0}^{T} H_{u} d S_{u} \geq 0\right)=1 \quad \Longrightarrow \quad \mathbb{P}\left(\int_{0}^{T} H_{u} d S_{u}=0\right)=1
$$

REMARK 4.3. Let $\theta \in \mathbb{L}(\mathbb{R})$. Due to Theorem 4.2, it is clear that the structure condition $(S C)$ is necessary for the price process $P^{\theta}$ in order to admit an equivalent local martingale measure. Hence, we start looking for sufficient conditions that ensure that $P^{\theta}$ satisfies (SC).

The next lemma highlights that it is natural to use the definition of $P^{\theta}$ to find conditions that ensure the existence of an equivalent local martingale measure. In 
the following, we denote by $\operatorname{TV}(Y)_{T}$ the total variation of the process $Y$ on $[0, T]$. Moreover, $\underset{u c p}{\longrightarrow}$ and $\underset{\mathcal{S M}}{\longrightarrow}$ indicate the convergence in the ucp-topology and the semimartingale topology, respectively.

LEMMA 4.4. Suppose that Assumption II holds and let $\left(\theta^{n}\right)_{n \geq 1} \subset \mathbf{S}(\mathbb{R})$. Besides, let $\theta \in \mathbb{L}(\mathbb{R}), S(\vartheta, \cdot)=S(\vartheta, 0)+M^{\vartheta}+\int \lambda^{\vartheta} d\left[M^{\vartheta}\right]$, and let

$$
F_{t}\left(\theta^{n}\right)=F_{0}\left(\theta^{n}\right) \mathbf{1}_{\{0\}}(t)+\sum_{i=0}^{m_{n}} F_{\tau_{i}^{n}+}\left(\theta^{n}\right) \mathbf{1}_{\left(\tau_{i}^{n}, \tau_{i+1}^{n}\right]}(t), \quad n \geq 1,
$$

be representations of $\left(F\left(\theta^{n}\right)\right)_{n \geq 1} \subset \mathbf{S}\left(\mathbb{R}^{d}\right)$. The following statements hold:

1. The canonical decompositions of $\left(P^{\theta^{n}}\right)_{n \geq 1}$ can be written as

$$
P^{\theta^{n}}=\int S\left(F_{S}\left(\theta^{n}\right), d s\right)=S\left(F_{0}\left(\theta^{n}\right), 0\right)+M^{n}+\int \lambda^{(n)} d\left[M^{n}\right],
$$

where $\int_{0}^{T}\left(\lambda_{u}^{(n)}\right)^{2} d\left[M^{n}\right]_{u}<\infty \mathbb{P}$-a.s. and

$$
\begin{aligned}
M_{t}^{n} & =\sum_{i=0}^{m_{n}}\left(M_{\tau_{i+1}^{n} \wedge t}^{F_{\tau_{i}^{n}+}\left(\theta^{n}\right)}-M_{\tau_{i}^{n} \wedge t}^{F_{\tau_{i}^{n}+}\left(\theta^{n}\right)}\right), \\
\lambda_{t}^{(n)} & =\sum_{i=0}^{m_{n}} \lambda_{t}^{F_{\tau_{i}^{n}+}\left(\theta^{n}\right)} \mathbf{1}_{\left(\tau_{i}^{n}, \tau_{i+1}^{n}\right]}(t) .
\end{aligned}
$$

2. If $\bar{S}_{0}+M+A$ denotes the canonical decomposition of $P^{\theta}$, where $M$ is a continuous local martingale and $M_{0}=0$, then $\theta^{n} \underset{u c p}{\longrightarrow} \theta$ ensures that

$$
M^{n} \underset{\mathcal{S M}}{\longrightarrow} M \text { and }\left[M^{n}\right] \underset{\mathcal{S M}}{\longrightarrow}[M] \text { and } \int \lambda^{(n)} d\left[M^{n}\right] \underset{\mathcal{S M}}{\longrightarrow} A
$$

3. In 2. there exists a subsequence (still indexed by $n$ ) such that $\mathbb{P}$-a.s.

$$
\operatorname{TV}\left(\int \lambda^{(n)} d\left[M^{n}\right]-A\right)_{T} \longrightarrow 0 .
$$

If $d=1$ and $F=i d$, then the statements also hold for $\left(\theta^{n}\right)_{n \geq 1} \subset \mathbf{S}^{e}(\mathbb{R})$.

REMARK 4.5. The lemma is the key tool to check whether or not $P^{\theta}$ satisfies $(S C)$. It allows us to use results from measure theory that guarantee the convergence of Lebesgue-Stieltjes integrals as in (18) to a limit of the form $\int \lambda d[M]$; see, for example, [8], 4.7.132-4.7.133. These results usually assume pointwise convergence of the integrands $\left(\lambda^{(n)}\right)_{n \geq 1}$. However, at this point, we do not know $a$ priori whether or not the convergence of $\left(\theta^{n}\right)_{n \geq 1}$ in the $u c p$-topology implies the pointwise convergence of the integrands $\left(\lambda^{(n)}\right)_{n \geq 1}$. 
The next theorem provides a sufficient condition for $P^{\theta}$ to satisfy (SC) without assuming pointwise convergence of $\left(\lambda^{(n)}\right)_{n \geq 1}$.

THEOREM 4.6. Suppose that Assumption II holds, let $\left(\theta^{n}\right)_{n \geq 1} \subset \mathbf{S}(\mathbb{R})$, $\theta \in \mathbb{L}(\mathbb{R})$ such that $\theta^{n} \longrightarrow \theta$ in the ucp-topology. Recall the notation of Lemma 4.4 and suppose that

$$
\liminf _{n \longrightarrow \infty} \int_{0}^{T}\left(\lambda_{u}^{(n)}\right)^{2} d\left[M^{n}\right]_{u}<\infty
$$

holds $\mathbb{P}$-a.s. Then the price process $P^{\theta}$ satisfies the structure condition (SC).

The following corollary is an immediate consequence of the above theorem.

COROLLARY 4.7. If there exists a sequence of stopping times $\left(R_{m}\right)_{m \geq 1} \uparrow T$ $\mathbb{P}$-a.s. and $\left(C_{m}\right)_{m \geq 1} \subset L^{2}(\mathbb{P})$ such that $\mathbb{P}\left(R_{m}=T\right) \longrightarrow 1$ for $m \longrightarrow \infty$ and

$$
\liminf _{n \longrightarrow \infty} \int_{0}^{R_{m}}\left(\lambda_{u}^{(n)}\right)^{2} d\left[M^{n}\right]_{u} \leq C_{m}
$$

holds for $m \geq 1 \mathbb{P}$-a.s., then $P^{\theta}$ satisfies the structure condition (SC).

Next, we show that (19) might depend on the approximating sequence.

EXAMPLE 4.8. Let $F=i d, W$ be a Brownian motion and $\operatorname{set} S(\vartheta, t)=W_{t}+$ $\vartheta t$. For $n \geq 1$, choose $\theta^{n}=\lambda^{n}=n \mathbf{1}_{\left(0, n^{-3 / 2}\right]}$. On the one hand, $\lambda^{n} \longrightarrow 0$ a.s. and in $L^{1}$. Therefore, we get $P^{\theta^{n}}=\int S\left(\theta^{n}, d s\right) \rightarrow W$ in the semimartingale topology. Although the limit satisfies $(S C)$ with $\lambda \equiv 0$, the assumption of Theorem 4.6 does not hold. Indeed, we have $\int_{0}^{T}\left|\lambda_{u}^{n}\right|^{2} d u=\sqrt{n}$. On the other hand, $\tilde{\theta}^{n}=1 / n \longrightarrow 0$ and satisfies (19).

Due to Theorem 4.2, the structure condition is a necessary condition for the existence of an equivalent local martingale measure for the discounted price process $P^{\theta}$, where its canonical decomposition is given by

$$
P^{\theta}=\bar{S}_{0}+M+\int \lambda d[M] .
$$

A candidate for a martingale measure is now given via the density process

$$
\mathcal{E}\left(-\int \lambda d M\right)=\exp \left(-\int \lambda d M-\frac{1}{2} \int \lambda^{2} d[M]\right) .
$$

This stochastic exponential is a strictly positive local $\mathbb{P}$-martingale and, therefore, a $\mathbb{P}$-supermartingale. It is well known that $\mathbb{E}\left[\mathcal{E}\left(-\int \lambda d M\right)_{T}\right]=1$ if and only if the stochastic exponential is a true martingale. If it were a true martingale, $d \mathbb{Q}=\mathcal{E}\left(-\int \lambda d M\right)_{T} d \mathbb{P}$ defines an equivalent local martingale measure for $P^{\theta}$. However, in general $\mathcal{E}\left(-\int \lambda d M\right)$ is not necessarily a true martingale; see [30]. 
The following proposition gives a sufficient condition for $\mathcal{E}\left(-\int \lambda d M\right)$ being a true martingale. Hence, it provides a sufficient condition for the existence of an equivalent local martingale measure for the price process $P^{\theta}$ if the large trader uses a general strategy $\theta \in \mathbb{L}(\mathbb{R})$.

PROPOSITION 4.9. Let $\theta \in \mathbb{L}(\mathbb{R})$ be a large trader strategy. Moreover, denote by $\left(\theta^{n}\right)_{n \geq 1} \subset \mathbf{S}(\mathbb{R})$ a sequence of strategies such that $\theta^{n} \longrightarrow \theta$ in the ucptopology. Recall the notation of Lemma 4.4 and suppose the following:

1. $\mathcal{E}\left(-\int \lambda^{n} d M^{n}\right)$ are true martingales for all $n \geq 1$.

2. The family $\left(\mathcal{E}\left(-\int \lambda^{n} d M^{n}\right)_{T}\right)_{n \geq 1}$ is uniformly integrable.

3. $\left[\int \lambda^{n} d M^{n}-\int \lambda d M\right]_{T} \longrightarrow 0$ in probability.

Then $\mathcal{E}\left(-\int \lambda d M\right)$ is a true martingale. Hence, $d \mathbb{Q}=\mathcal{E}\left(-\int \lambda d M\right)_{T} d \mathbb{P}$ is an equivalent local martingale measure for the discounted price process $P^{\theta}$.

We end this section with an example. It is an application of Corollary 4.7.

EXAmple 4.10. Due to Lemma A.2, we can find $\left(\theta^{n}\right)_{n \geq 1} \subset \mathbf{S}^{e}(\mathbb{R})$, where

$$
F_{t}\left(\theta^{n}\right)=\theta^{n}(t)=\theta_{-1} \mathbf{1}_{\{0\}}(t)+\sum_{i=0}^{m_{n}} \theta_{i}^{n} \mathbf{1}_{\left(\tau_{i}^{n}, \tau_{i+1}^{n}\right]}(t), \quad n \geq 1,
$$

and a sequence $\left(R_{m}\right)_{m \geq 1}$ of stopping times such that $\left\{\left(\theta^{n}\right)^{R_{m}}, \theta^{R_{m}}\right\}$ is uniformly bounded, $\mathbb{P}\left(R_{m}=T\right) \longrightarrow 1$ for $m \longrightarrow \infty$, and $\theta^{n} \rightarrow \theta \in \mathbb{L}(\mathbb{R})$ in the ucptopology. Since $F=i d$, Lemma 4.4 and Remark 4.1 ensure that the canonical decomposition of $P^{\theta^{n}}$ is given by

$$
P^{\theta^{n}}=S\left(\theta_{-1}^{n}, 0\right)+M^{n}+\int \lambda^{(n)} d\left[M^{n}\right]
$$

where $M^{n}$ and $\lambda^{(n)}$ are defined in (17).

1. Stochastic differential equations: In the setting of Example 2.12, the local martingales $M^{n}$ as well as the $\lambda^{(n)}$ are given by

$$
\begin{aligned}
M_{t}^{n} & =\sum_{i=0}^{m_{n}} \sigma\left(S\left(\theta_{i}^{n}, \tau_{i}^{n}\right)\right)\left(W_{\tau_{i+1}^{n} \wedge t}-W_{\tau_{i}^{n} \wedge t}\right), \\
\lambda_{t}^{(n)} & =\sum_{i=0}^{m_{n}} \lambda_{t}^{\theta_{i}^{n}} \mathbf{1}_{\left(\tau_{i}^{n}, \tau_{i+1}^{n}\right]}(t)=\sum_{i=0}^{m_{n}} \frac{b^{\theta_{i}^{n}}\left(S\left(\theta_{i}^{n}, \tau_{i}^{n}\right)\right)}{\sigma^{2}\left(S\left(\theta_{i}^{n}, \tau_{i}^{n}\right)\right)} \mathbf{1}_{\left(\tau_{i}^{n}, \tau_{i+1}^{n}\right]}(t) .
\end{aligned}
$$

Since

$$
\begin{aligned}
\int_{0}^{T}\left(\lambda_{u}^{(n)}\right)^{2} d\left[M^{n}\right]_{u} & =\int_{0}^{T}\left(\sum_{i=0}^{m_{n}} \frac{b^{\theta_{i}^{n}}\left(S\left(\theta_{i}^{n}, \tau_{i}^{n}\right)\right)}{\sigma^{2}\left(S\left(\theta_{i}^{n}, \tau_{i}^{n}\right)\right)} \mathbf{1}_{\left(\tau_{i}^{n}, \tau_{i+1}^{n}\right]}(u)\right)^{2} d\left[M^{n}\right]_{u} \\
& =\sum_{i=0}^{m_{n}}\left(\frac{b^{\theta_{i}^{n}}\left(S\left(\theta_{i}^{n}, \tau_{i}^{n}\right)\right)}{\sigma^{2}\left(S\left(\theta_{i}^{n}, \tau_{i}^{n}\right)\right)}\right)^{2}\left(\tau_{i+1}^{n}-\tau_{i}^{n}\right)
\end{aligned}
$$


the assumptions as well as the special choice of $\left(\theta^{n}\right)_{n \geq 1}$ and $\left(R_{m}\right)_{m \geq 1}$ ensure that we can apply Corollary 4.7. Therefore, $P^{\theta}$ satisfies $(S C)$.

As by assumption $\sigma>\epsilon$, the ratio $b^{\vartheta}(x) / \sigma^{2}(x)$ is again continuous in both arguments and nondecreasing in $\vartheta$. The same arguments as in Lemma A.10 ensure that $b^{\vartheta}(x) / \sigma^{2}(x)$ is jointly continuous. If, in addition, the family $\left\{\left(\theta^{n}\right)_{n \geq 1}, \theta\right\}$ is uniformly bounded and there exists a continuous function $f: \mathbb{R} \longrightarrow \mathbb{R}$ and a constant $c>0$ such that

$$
\left|\frac{b^{\vartheta}(x)}{\sigma^{2}(x)}\right| \leq c f(\vartheta), \quad \forall(\vartheta, x) \in \mathbb{R} \times \mathbb{R},
$$

then there exists $K>0$ such that

$$
\int_{0}^{T} \lambda_{u}^{2} d[M]_{u} \leq c^{2} \liminf _{n \longrightarrow \infty} \sum_{i=0}^{m_{n}} f^{2}\left(\theta_{i}^{n}\right)\left(\tau_{i+1}^{n}-\tau_{i}^{n}\right) \leq c^{2} K^{2} T .
$$

On that account, Novikov's condition ensures that $\mathcal{E}\left(-\int \lambda d M\right)$ is a true martingale. Thus, $d \mathbb{Q}=\mathcal{E}\left(-\int \lambda d M\right)_{T} d \mathbb{P}$ is an equivalent local martingale measure for $P^{\theta}$.

2. Reaction-diffusion setting: Similar calculations as above show that in the setting of Example 2.13 we have

$$
\begin{aligned}
M_{t}^{n} & =\sum_{i=0}^{m_{n}} \frac{\partial}{\partial x} \psi\left(\tau_{i}^{n}, W_{\tau_{i}^{n}}, \theta_{i}^{n}\right)\left(W_{\tau_{i+1}^{n} \wedge t}-W_{\tau_{i}^{n} \wedge t}\right), \\
\lambda_{t}^{(n)} & =\sum_{i=0}^{m_{n}} \frac{\left(\frac{\partial}{\partial t}+\frac{1}{2} \frac{\partial^{2}}{\partial x^{2}}\right) \psi\left(\tau_{i}^{n}, W_{\left.\tau_{i}^{n}, \theta_{i}^{n}\right)}\right.}{\left(\frac{\partial}{\partial x} \psi\left(\tau_{i}^{n}, W_{\tau_{i}^{n}}, \theta_{i}^{n}\right)\right)^{2}} \mathbf{1}_{\left(\tau_{i}^{n}, \tau_{i+1}^{n}\right]}(t)
\end{aligned}
$$

and

$$
\begin{aligned}
& \int_{0}^{T}\left(\lambda_{u}^{(n)}\right)^{2} d\left[M^{n}\right]_{u} \\
& \quad=\sum_{i=0}^{m_{n}}\left(\frac{\left(\frac{\partial}{\partial t}+\frac{1}{2} \frac{\partial^{2}}{\partial x^{2}}\right) \psi\left(\tau_{i}^{n}, W_{\tau_{i}^{n}}, \theta_{i}^{n}\right)}{\frac{\partial}{\partial x} \psi\left(\tau_{i}^{n}, W_{\tau_{i}^{n}}, \theta_{i}^{n}\right)}\right)^{2}\left(\tau_{i+1}^{n}-\tau_{i}^{n}\right) .
\end{aligned}
$$

For general reaction functions $\psi$, it is plausible that one has to impose certain conditions on the fraction above to ensure that the condition of Theorem 4.6 is satisfied. However, for the reaction function $\psi(t, x, \vartheta)=\exp (\sigma x+\kappa \vartheta t)$, where $\sigma, \kappa>0$, we get

$$
\int_{0}^{T}\left(\lambda_{u}^{(n)}\right)^{2} d\left[M^{n}\right]_{u}=\sum_{i=0}^{m_{n}}\left(\frac{\kappa}{\sigma} \theta_{i}^{n}+\frac{1}{2} \sigma\right)^{2}\left(\tau_{i+1}^{n}-\tau_{i}^{n}\right) .
$$


Due to the special choice of $\left(\theta^{n}\right)_{n \geq 1}$ and $\left(R_{m}\right)_{m \geq 1}$, we may apply Corollary 4.7 and conclude that $P^{\theta}$ satisfies the structure condition $(S C)$. If the sequence is uniformly bounded by some constant $K>0$, we get

$$
\int_{0}^{T} \lambda_{u}^{2} d[M]_{u} \leq 2 T\left(\frac{\kappa^{2} K^{2}}{\sigma^{2}}+\frac{\sigma^{2}}{4}\right) .
$$

Again, Novikov's condition ensures that $d \mathbb{Q}=\mathcal{E}\left(-\int \lambda d M\right)_{T} d \mathbb{P}$ defines an equivalent local martingale measure for $P^{\theta}$.

\section{Utility maximization: Description of new phenomena arising in a large} trader setting. When it comes to modelling the effects on the price process caused by the large trader's actions, the most crucial question is: "What is the large trader's motivation to trade?" Here, we consider a large trader seeking to maximize her expected utility from terminal wealth.

In order to formulate the utility maximization problem, we first have to provide an admissibility concept for the large trader. On the one hand, we think that this definition should coincide with at least one generic definition of admissibility in the small trader setting if the large trader does not influence the price process. In this case, she is in fact a small trader. On the other hand, the most popular admissibility concept, the $a$-admissibility, where the wealth process is bounded from below by a finite credit line $a$, is not tractable in our setting. Although this admissibility concept has a clear economic interpretation, it might lead to a prohibition of all constant large trader strategies (except $\theta \equiv 0$ ); see Remark 5.4 below. As simple strategies form the core of our model, this would be highly unsatisfactory. Here, we use a modified version of the concept suggested by Biagini and Sirbu [7]. These authors suggest (in a small trader setting) to consider those strategies that allow for a loss control of the associated (real) wealth process by a martingale under the historical measure. Taking these considerations into account, we end up with the following definition of an admissible large trader strategy.

DEFINITION 5.1. Let $\left((S(\vartheta, \cdot))_{\vartheta \in \mathbb{R}^{d}}, F, C, \theta\right)$ be a minimal large trader market model. The large trader strategy $\theta \in \mathbb{L}(\mathbb{R})$ is called admissible if there exists a strictly positive $\mathbb{P}$-martingale $L^{\theta}$ such that

$$
V(\theta) \geq-L^{\theta}
$$

holds up to indistinguishability. We call $L^{\theta}$ a loss control of the strategy $\theta$.

In order to formulate the utility maximization problem, we need the following definition. It traces back to $[5,6]$.

DEFINITION 5.2. Let $u: \mathbb{R} \longrightarrow \mathbb{R}$ be a strictly concave, increasing and twice continuously differentiable function that satisfies the Inada conditions

$$
u^{\prime}(-\infty):=\lim _{x \longrightarrow-\infty} u^{\prime}(x)=-\infty \quad \text { and } \quad u^{\prime}(+\infty):=\lim _{x \longrightarrow+\infty} u^{\prime}(x)=0 .
$$


An admissible large trader strategy $\theta \in \mathbb{L}(\mathbb{R})$ is called $u$-compatible for $\alpha>0$ if there exists a loss control $L^{\theta}$ that satisfies

$$
\mathbb{E}\left[u\left(-\alpha L_{T}^{\theta}\right)\right]>-\infty .
$$

For $\alpha>0$, we denote by $\mathcal{H}^{\alpha}$ the set of all admissible large trader strategies that are $u$-compatible for $\alpha$. Furthermore, we set $\mathcal{H}:=\bigcap_{\alpha>0} \mathcal{H}^{\alpha}$.

We analyse the utility maximization problem of a large trader in a basic setting. Despite its rather simple structure, it highlights new phenomena that are not present in the classical utility maximization theory for small traders. At this point, we want to emphasize that these phenomena show up despite the fact that there exists an equivalent local martingale measure for the real wealth process of the large trader. Besides, in some cases the real wealth process is even a supermartingale under this measure. These phenomena are a consequence of the nonlinear structure of the wealth process.

Let $d=1, F=i d$, and define the primal family $(S(\vartheta, \cdot))_{\vartheta \in \mathbb{R}}$ by

$$
S(\vartheta, t):=S_{0}+\mu(\vartheta)[M]_{t}+\sigma(\vartheta) M_{t}, \quad t \in[0, T],
$$

where $M$ is a continuous and square-integrable martingale starting in zero a.s. and having a deterministic quadratic variation $[M]$. Besides, let $S_{0} \in \mathbb{R}$, $\mu, \sigma \in C^{2}(\mathbb{R}, \mathbb{R})$, such that $\mu$ vanishes whenever $\sigma$ is zero. Hence, (NA-II) holds. Due to Theorem 2.10, $(S(\vartheta, \cdot))_{\vartheta \in \mathbb{R}}$ satisfies (P-II). Furthermore, let $C: \mathbf{S}(\mathbb{R}) \longrightarrow$ $\mathcal{V}_{\geq 0}$ be a cost function that maps constant strategies to zero. For an admissible large trader strategy $\theta \in \mathbb{L}(\mathbb{R})$, the price process and the large trader real wealth process are given by

$$
\begin{aligned}
P^{\theta} & =S_{0}+\int \mu(\theta) d[M]+\int \sigma(\theta) d M, \\
V(\theta) & =V_{0}^{\theta}+\int \theta \mu(\theta) d[M]+\int \theta \sigma(\theta) d M-C(\theta) .
\end{aligned}
$$

For $\alpha>0$, we consider the exponential utility maximization problem

$$
\sup _{\theta \in \mathcal{H}^{1}} \mathbb{E}\left[u\left(V_{T}(\theta)\right)\right]
$$

where $u(x)=1-e^{-\alpha x}$. Next, we provide a partial characterization of $\mathcal{H}$.

LEMMA 5.3. Let $(S(\vartheta, \cdot))_{\vartheta \in \mathbb{R}}$ be given by (21) and let $u(x)=1-e^{-\alpha x}$ for $\alpha>0$. If $\theta \in b \mathbb{L}(\mathbb{R})$ and $C(\theta)$ are bounded, then $\theta \in \mathcal{H}$. Moreover, for all $\alpha>0$ the process $L^{\theta}$ is an $u$-compatible loss control for $\theta$, where

$$
L_{t}^{\theta}:=\mathbb{E}\left[\sup _{s \leq T}\left|V_{s}(\theta)\right| \mid \mathcal{F}_{t}\right]
$$

REMARK 5.4. The lemma highlights that the concept of $a$-admissibility is not suitable for our large trader setting. Indeed, if $M$ in (21) is a Brownian motion, it would prohibit any constant large trader strategy except $\theta=0$. 
Recall Definition 3.7 of tame strategies and define

$$
\Phi:=\{\theta \in b \mathbb{L}(\mathbb{R}) \mid \theta \text { is a tame admissible large trader strategy }\} .
$$

Due to Novikov's condition, we can define probability measures $\mathbb{Q}^{\theta} \sim \mathbb{P}$ via

$$
\text { (23) } \frac{d \mathbb{Q}^{\theta}}{d \mathbb{P}}:=\exp \left(-\alpha \int_{0}^{T} \theta_{u} \sigma\left(\theta_{u}\right) d M_{u}-\frac{\alpha^{2}}{2} \int_{0}^{T} \theta_{u}^{2} \sigma^{2}\left(\theta_{u}\right) d[M]_{u}\right), \quad \forall \theta \in \Phi \text {. }
$$

Now we can rewrite the utility maximization problem (22) for all $\theta \in \Phi$ as

$$
\mathbb{E}\left[u\left(V_{T}(\theta)\right)\right]=1-e^{-\alpha V_{0}^{\theta}} \mathbb{E}_{\mathbb{Q}^{\theta}}\left[e^{-\alpha \int_{0}^{T} p\left(\theta_{u}\right) d[M]_{u}}\right],
$$

where

$$
p(x):=x \mu(x)-\frac{\alpha}{2} x^{2} \sigma^{2}(x), \quad x \in \mathbb{R} .
$$

After these preliminary observations, we first discuss the reduced problem

$$
\sup _{\theta \in \Phi} \mathbb{E}\left[u\left(V_{T}(\theta)\right)\right] \text {. }
$$

As we will see below, two different scenarios might happen. In the first scenario, the so-called stable regime, we can find at least one optimal strategy. These optimal strategies are constant. In the second scenario, the unstable regime, the presence of the large trader completely destabilizes the market. This is due to the fact that it is optimal for the large trader to buy/sell as many shares as possible to maximize her expected utility.

REMARK 5.5. It will turn out that the existence of an optimal strategy boils down to the existence of a maximum of the function $p$. The following observations show that the market is stable if and only if $p$, defined in (25), attains at least one maximum:

1. Stable regime: Suppose that $p$ has at least one maximum $\vartheta^{*} \in \mathbb{R}$. Then

$$
\mathbb{E}\left[u\left(V_{T}(\theta)\right)\right]=1-e^{-\alpha\left(V_{0}^{\theta}+p\left(\vartheta^{*}\right)[M]_{T}\right)} \mathbb{E}_{\mathbb{Q}^{\theta}}\left[e^{-\alpha \int_{0}^{T}\left(p\left(\theta_{u}\right)-p\left(\vartheta^{*}\right)\right) d[M]_{u}}\right]
$$

is equivalent to (24) for all $\theta \in \Phi$. Since $\mathbb{P}\left(p(\theta)-p\left(\vartheta^{*}\right) \leq 0, \forall \theta \in \Phi\right)=1$, it follows that $\vartheta^{*}$ is an optimal strategy and

$$
\sup _{\theta \in \Phi} \mathbb{E}\left[u\left(V_{T}(\theta)\right)\right]=1-\exp \left(-\alpha\left(V_{0}^{\theta}+p\left(\vartheta^{*}\right)[M]_{T}\right)\right) .
$$

2. Unstable regime: Suppose that $p$ has no maximum. Due to the continuity of $p$, we can find a sequence $\left(\vartheta_{n}\right)_{n \in \mathbb{N}} \subset \Phi$ of constant strategies such that

$$
\sup _{\vartheta \in \mathbb{R}} p(\vartheta)= \begin{cases}\lim _{n \rightarrow \infty} p\left(\vartheta_{n}\right)=: p^{*} \in \mathbb{R} & \text { if } p \text { is bounded from above, } \\ +\infty & \text { else. }\end{cases}
$$


Keeping this in mind, it follows that

$$
\sup _{\theta \in \Phi} \mathbb{E}\left[u\left(V_{T}(\theta)\right)\right]= \begin{cases}1-e^{-\alpha\left(V_{0}^{\theta}+p^{*}[M]_{T}\right)} & \text { if } p \text { is bounded from above, } \\ 1 & \text { else. }\end{cases}
$$

Since continuous functions attain their extreme points on compact intervals, it is clear that $\vartheta_{n} \longrightarrow \pm \infty$. Obviously, $\vartheta_{n} \longrightarrow+\infty$ means that the large trader tries to buy as many shares as possible in order to reach her maximal expected utility from terminal wealth. $\vartheta_{n} \longrightarrow-\infty$ means that she achieves her goal by short selling. Thus, there is no optimal strategy $\theta \in \Phi$. Such strategies lead to exploding or collapsing prices and destabilize the market.

We collect the above results in the following proposition.

PROPOSITION 5.6. Under the assumptions made above, we have

$$
\sup _{\theta \in \Phi} \mathbb{E}\left[u\left(V_{T}(\theta)\right)\right]=\sup _{\theta \in \mathcal{H}^{1}} \mathbb{E}\left[u\left(V_{T}(\theta)\right)\right] .
$$

Furthermore, either of the following statements hold:

1. Stable regime: There exists at least one solution to the utility maximization problem (22) if and only if the function p defined in (25) attains at least one maximum. The set of optimal strategies contains only constant strategies and (if considered as subset of $\mathbb{R}$ ) coincides with the set of maxima of the function $p$. If $\vartheta^{*} \in \mathbb{R}$ is an optimal strategy, the value of the utility maximization problem (22) is given by

$$
\sup _{\theta \in \Phi} \mathbb{E}\left[u\left(V_{T}(\theta)\right)\right]=1-\exp \left(-\alpha\left(V_{0}^{\theta}+p\left(\vartheta^{*}\right)[M]_{T}\right)\right) .
$$

2. Unstable regime: There is no optimal trading strategy in $\mathcal{H}^{1}$. Moreover, by maximizing the expected utility from terminal wealth, the large trader destabilizes the market since the prices either explode or collapse. Here, her utility maximization efforts lead to

$$
\sup _{\theta \in \Phi} \mathbb{E}\left[u\left(V_{T}(\theta)\right)\right]= \begin{cases}1-e^{-\alpha\left(V_{0}^{\theta}+p^{*}[M]_{T}\right)} & p \text { is bounded from above, } \\ 1 & \text { else. }\end{cases}
$$

REMARK 5.7. 1. Due to Proposition 5.6, it is easy to find an example in which the necessary condition is not sufficient. Choose for instance $\mu(x)=x$ and $\sigma(x)=x^{2}$. Then $\vartheta^{*}=0$ satisfies the necessary condition $p^{\prime}\left(\vartheta^{*}\right)=0$. As $p^{\prime \prime}\left(\vartheta^{*}\right)=2, \vartheta^{*}$ is a local minimum of $p$. Due to Proposition 5.6, this constant trading strategy is not optimal.

2. Note that in the stable regime there exists an equivalent local martingale measure for the real wealth process. Furthermore, the "destabilization" of the market 
in the unstable regime can be achieved by a sequence of large trader strategies such that for all of these wealth processes there exists an equivalent local martingale measure. Finally, if $M$ in (21) is a Brownian motion, the corresponding real wealth processes are also supermartingales under the equivalent local martingale measure.

3. Recall our discussion on "The model chooses the strategies" in Section 3.2. In the context of utility maximization, the stable regime is an example in which the model chooses its strategies.

EXAMPLE 5.8 (Illiquid Bachelier model). Define $(S(\vartheta, \cdot))_{\vartheta \in \mathbb{R}}$ by

$$
S(\vartheta, t)=S_{0}+(\mu+\kappa \vartheta) t+\sigma W_{t},
$$

where $W$ is a Brownian motion and $\mu, \kappa, \sigma$ are positive parameters. The filtration is supposed to be the smallest one fulfilling the usual conditions and containing the one generated by $W$.

To analyse the model, we apply the same arguments as in Proposition 5.6. The function $p$ defined in (25) is a polynomial of order 2 . In particular, we find that for $\alpha \sigma^{2} \neq 2 \kappa$,

$$
p(x)=-\frac{\alpha \sigma^{2}-2 \kappa}{2}\left(x-\frac{\mu}{\alpha \sigma^{2}-2 \kappa}\right)^{2}+\frac{\mu^{2}}{2\left(\alpha \sigma^{2}-2 \kappa\right)} .
$$

Clearly,

$$
\vartheta^{*}=\frac{\mu}{\alpha \sigma^{2}-2 \kappa}
$$

satisfies the necessary condition $p^{\prime}\left(\vartheta^{*}\right)=0$. Therefore, utility maximization relies on the stability condition $p^{\prime \prime}\left(\vartheta^{*}\right)<0$, which is equivalent to $2 \kappa<\alpha \sigma^{2}$.

1. Stable regime $2 \kappa<\alpha \sigma^{2}$ : The strategy $\vartheta^{*}$ performs best among all strategies. Moreover, it is the only admissible strategy which fulfils the necessary optimality condition. If the initial value equals 0 and the large trader chooses the strategy $\vartheta^{*}$, she gains an expected utility of

$$
1-\exp \left(-\frac{\alpha \mu^{2} T}{2\left(\alpha \sigma^{2}-2 \kappa\right)}\right)
$$

We now want to compare the expected utility of the large trader with the optimal utility in the classical Merton problem in this stable regime. Consider the case where we face a hypothetical small investor with the same utility function and initial wealth 0 , and with given price process $P^{\theta}$. Given that the large trader is present in the market and behaves rationally, that is, chooses the constant strategy $\theta=\vartheta^{*}$, it results that the small trader would choose a constant strategy as well, 
namely

$$
\psi\left(\vartheta^{*}\right)=\frac{\mu+\kappa \vartheta^{*}}{\alpha \sigma^{2}}
$$

His expected utility in that case would be

$$
1-e^{-\frac{\alpha^{2} \psi^{2}\left(\vartheta^{*}\right) \sigma^{2} T}{2}}=1-\exp \left(-\frac{\alpha \mu^{2} T}{2\left(\alpha \sigma^{2}-2 \kappa\right)}\left(1+\frac{\kappa^{2}}{\alpha \sigma^{2}\left(\alpha \sigma^{2}-2 \kappa\right)}\right)\right) .
$$

Therefore, the small investor would achieve a higher expected utility. If there were no large trader around, that is, $\theta=0$, the small trader would hold an optimal portfolio of

$$
\psi(0)=\frac{\mu}{\alpha \sigma^{2}}
$$

stocks, and his expected utility in that case is

$$
1-\exp \left(-\frac{\mu^{2} T}{2 \sigma^{2}}\right)=1-\exp \left(-\frac{\alpha \mu^{2} T}{2\left(\alpha \sigma^{2}-2 \kappa\right)}\left(1-\frac{2 \kappa}{\alpha \sigma^{2}}\right)\right) .
$$

Accordingly, the absence of the large trader is not beneficial for the small trader as it reduces his expected utility.

In [4], Bank and Baum compare the utility maximization problem of the large trader to the utility maximization problem of the small trader in the market $P^{0}$. Under [4], Assumption 4, which requires that $S(\vartheta, t)=S(\vartheta, 0)+\int_{0}^{t} p_{u}^{\vartheta} d S(\vartheta, u)$ for all $\vartheta \in \mathbb{R}$, they prove that the utility maximization efforts of both traders lead to the same value. However, our primal family does not satisfy [4], Assumption 4. Moreover, the small trader achieves only a lower expected utility (in the model $P^{0}$ ) compared to the large trader that maximizes her expected utility. The only exception would be the case $\mu=0$.

2. Unstable regime:

(a) Case $2 \kappa>\alpha \sigma^{2}$ : In this case, $\vartheta^{*}$ is the minimum of the function $p$. Thus, the strategy $\vartheta^{*}$ performs worst among all strategies, while the expected utility grows with $|\vartheta|$ up to the maximum value. This can be interpreted in a way that the impact on the drift is so substantial that the large investor buys as many shares as possible.

(b) Case $2 \kappa=\alpha \sigma^{2}$ : In this critical case, $p$ defined in (25) is given by $p(x)=$ $\mu x$. Hence, the result depends on $\mu$. If $\mu=0$, all strategies perform equally as the investor always gets the expected utility of the zero strategy. For $\mu \neq 0$ she can, like above, achieve expected utility arbitrarily close to the maximum value of one. Yet, now her stake has to have the right sign, depending on the sign of $\mu$.

REMARK 5.9. This example is not included in the model class studied by Kühn [24] whose Assumption 2.1 ("Largeness is not favourable") implies that the drift is nonincreasing in $\vartheta$. 


\section{Proofs.}

Proof of Proposition 2.8. For $\vartheta \in \mathbb{R}^{d}$, let $Z^{\vartheta}$ denote the density process of a local martingale measure $\mathbb{Q}^{\vartheta}$ for $S(\vartheta, \cdot)$ which exists due to (NA-II). As $F(\tilde{\theta})=: \theta$ is a simple strategy, all the $\theta_{i}$ in the representation (3) assume only finitely many values denoted by $\left\{\vartheta_{i_{1}}, \ldots, \vartheta_{i_{m_{i}}}\right\}$. We may assume by localization that the $S(\vartheta, \cdot)$ are $\mathbb{Q}^{\vartheta}$-martingales for each $\vartheta$ from this finite set. Define probability measures $\mathbb{Q}^{\theta_{i}}, i \in\{0, \ldots, n\}$, by

$$
Z_{t}^{\theta_{i}}:=\left.\frac{d \mathbb{Q}^{\theta_{i}}}{d \mathbb{P}}\right|_{\mathcal{F}_{t}}:=c_{i} \sum_{j=1}^{m_{i}} Z_{t}^{\vartheta_{i j}} \mathbf{1}_{\left\{\theta_{i}=\vartheta_{i j}\right\}},
$$

where the $c_{i}$ are normalising constants. Then $\int S\left(\theta_{i}, d s\right)$ is a $\mathbb{Q}^{\theta_{i}}$-martingale for all $i \in\{0, \ldots, n\}$. Indeed, for each $i \in\{0, \ldots, n\}$ and $t \in[0, T]$, we set

$$
\begin{aligned}
\Delta_{t} S^{i} & :=S\left(\theta_{i}, \tau_{i+1} \wedge t\right)-S\left(\theta_{i}, \tau_{i} \wedge t\right), \\
\Delta_{t} S^{i, j} & :=S\left(\vartheta_{i j}, \tau_{i+1} \wedge t\right)-S\left(\vartheta_{i j}, \tau_{i} \wedge t\right) .
\end{aligned}
$$

Bayes' formula enables us to compute the conditional expectations under a measure change. Hence, for $i \in\{0, \ldots, n\}$ and $s<t$ we get

$$
\begin{aligned}
\mathbb{E}_{\mathbb{Q}^{\theta_{i}}}\left[\Delta_{t} S^{i} \mid \mathcal{F}_{S}\right] & =\frac{1}{Z_{s}^{\theta_{i}}} \mathbb{E}_{\mathbb{P}}\left[Z_{T}^{\theta_{i}} \Delta_{t} S^{i} \mid \mathcal{F}_{S}\right] \\
& =\frac{1}{Z_{s}^{\theta_{i}}} c_{i} \sum_{j=1}^{m_{i}} \mathbb{E}_{\mathbb{P}}\left[Z_{T}^{\vartheta_{i j}} \Delta_{t} S^{i} \mathbf{1}_{\left\{\theta_{i}=\vartheta_{i j}\right\}} \mid \mathcal{F}_{S}\right] \\
& =c_{i} \sum_{j=1}^{m_{i}} \frac{Z_{s}^{\vartheta_{i j}}}{Z_{S}^{\theta_{i}}} \mathbb{E}_{\mathbb{Q}^{\vartheta_{i j}}}\left[\Delta_{t} S^{i j} \mathbf{1}_{\left\{\theta_{i}=\vartheta_{i j}\right\}} \mid \mathcal{F}_{S}\right] .
\end{aligned}
$$

Moreover, for fixed $j \in\left\{1, \ldots, m_{i}\right\}$ we compute

$$
\begin{aligned}
\mathbb{E}_{\mathbb{Q}^{\vartheta_{i j}}}\left[\Delta_{t} S^{i j} \mathbf{1}_{\left\{\theta_{i}=\vartheta_{i j}\right\}} \mid \mathcal{F}_{S}\right]= & \mathbb{E}_{\mathbb{Q}^{\vartheta_{i j}}}\left[\Delta_{t} S^{i j} \mathbf{1}_{\left\{\theta_{i}=\vartheta_{i j}\right\}} \mathbf{1}_{\left\{s \geq \tau_{i}\right\}} \mid \mathcal{F}_{S}\right] \\
& +\mathbb{E}_{\mathbb{Q}^{\vartheta_{i j}}}\left[\Delta_{t} S^{i j} \mathbf{1}_{\left\{\theta_{i}=\vartheta_{i j}\right\}} \mathbf{1}_{\left\{s<\tau_{i}\right\}} \mid \mathcal{F}_{S}\right] .
\end{aligned}
$$

First, let us compute the first term on the right-hand side of (28). Due to (NA-II), we know that $\Delta_{t} S^{i j}$ are $\mathbb{Q}^{\vartheta_{i j}}$-martingales for all $j \in\left\{1, \ldots, m_{i}\right\}$. As $\theta_{i}$ is $\mathcal{F}_{\tau_{i}}$ measurable, we conclude that

$$
\begin{aligned}
\mathbb{E}_{\mathbb{Q}^{\vartheta_{i j}}}\left[\Delta_{t} S^{i j} \mathbf{1}_{\left\{\theta_{i}=\vartheta_{i j}\right\}} \mathbf{1}_{\left\{s \geq \tau_{i}\right\}} \mid \mathcal{F}_{S}\right] & =\mathbb{E}_{\mathbb{Q}^{\vartheta_{i j}}}\left[\Delta_{t} S^{i j} \mid \mathcal{F}_{S}\right] \mathbf{1}_{\left\{\theta_{i}=\vartheta_{i j}\right\}} \mathbf{1}_{\left\{s \geq \tau_{i}\right\}} \\
& =\Delta_{S} S^{i j} \mathbf{1}_{\left\{\theta_{i}=\vartheta_{i j}\right\}} \mathbf{1}_{\left\{s \geq \tau_{i}\right\}} .
\end{aligned}
$$


In the next step, we compute the second term on the right-hand side of (28). It equals zero. Indeed, by conditioning on $\mathcal{F}_{\tau_{i}}$ and using the tower property of conditional expectation, we get

$$
\begin{aligned}
\mathbb{E}_{\mathbb{Q}^{\vartheta^{i j}}} & {\left[\Delta_{t} S^{i j} \mathbf{1}_{\left\{\theta_{i}=\vartheta_{i j}\right\}} \mathbf{1}_{\left\{s<\tau_{i}\right\}} \mid \mathcal{F}_{S}\right] } \\
& =\mathbb{E}_{\mathbb{Q}^{\vartheta_{i j}}}\left[\mathbb{E}_{\mathbb{Q}^{\vartheta_{i j}}}\left[\Delta_{t} S^{i j} \mathbf{1}_{\left\{\theta_{i}=\vartheta_{i j}\right\}} \mathbf{1}_{\left\{s<\tau_{i}\right\}} \mid \mathcal{F}_{\tau_{i}}\right] \mid \mathcal{F}_{s}\right] \\
& =\mathbb{E}_{\mathbb{Q}^{\vartheta_{i j}}}\left[\mathbf{1}_{\left\{\theta_{i}=\vartheta_{i j}\right\}} \mathbf{1}_{\left\{s<\tau_{i}\right\}} \mathbb{E}_{\mathbb{Q}^{\vartheta_{i j}}}\left[\Delta_{t} S^{i j} \mid \mathcal{F}_{\tau_{i}}\right] \mid \mathcal{F}_{S}\right]
\end{aligned}
$$

Due to the martingale property of $S\left(\vartheta_{i j}, \cdot\right)$, we can conclude that

$$
\begin{aligned}
\mathbb{E}_{\mathbb{Q}^{\vartheta_{i j}}} & {\left[\mathbf{1}_{\left\{\theta_{i}=\vartheta_{i j}\right\}} \mathbf{1}_{\left\{s<\tau_{i}\right\}} \mathbb{E}_{\mathbb{Q}^{\vartheta_{i j}}}\left[\Delta_{t} S^{i j} \mid \mathcal{F}_{\tau_{i}}\right] \mid \mathcal{F}_{S}\right] } \\
= & \mathbb{E}_{\mathbb{Q}^{\vartheta_{i j}}}\left[\mathbf{1}_{\left\{\theta_{i}=\vartheta_{i j}\right\} \cap\left\{s<\tau_{i}\right\}} \Delta_{t \wedge \tau_{i}} S^{i j} \mid \mathcal{F}_{S}\right]=0 .
\end{aligned}
$$

Since $\Delta_{S} S^{i j} \mathbf{1}_{\left\{\theta_{i}=\vartheta_{i j}\right\}} \mathbf{1}_{\left\{s<\tau_{i}\right\}}$ is zero, the computations above ensure that

$$
\mathbb{E}_{\mathbb{Q}^{\vartheta_{i j}}}\left[\Delta_{t} S^{i j} \mathbf{1}_{\left\{\theta_{i}=\vartheta_{i j}\right\}} \mid \mathcal{F}_{S}\right]=\Delta_{S} S^{i j} \mathbf{1}_{\left\{\theta_{i}=\vartheta_{i j}\right\}}
$$

and (28) are equivalent. Summing up over $j$, we deduce from (27) that

$$
\mathbb{E}_{\mathbb{Q}^{\theta_{i}}}\left[\Delta_{t} S^{i} \mid \mathcal{F}_{S}\right]=\Delta_{s} S^{i}
$$

Hence, $\int S\left(\theta_{i}, d s\right)$ is a local martingale under $\mathbb{Q}^{\theta_{i}}$. Finally, we construct the density process $Z^{\theta}$ of $\mathbb{Q}^{\theta}$ on the whole time interval $[0, T]$ by concatenation,

$$
Z_{t}^{\theta}:=\prod_{i=0}^{n} \frac{Z_{t \wedge \tau_{i+1}}^{\theta_{i}}}{Z_{t \wedge \tau_{i}}^{\theta_{i}}}
$$

In particular, $\mathbb{Q}^{\tilde{\theta}}:=\mathbb{Q}^{\theta}$ is an equivalent local martingale measure for $P^{\tilde{\theta}}$.

Proof OF THEOREM 2.10. Without loss of generality, we assume that $S(\vartheta, 0)=0$ for all $\vartheta \in \mathbb{R}^{d}$. Due to Lemma A.10, Definition A.8 1 holds. To prove the second item, let $K>0$ and define the sequence of stopping times $\left(\tau_{n}^{K}\right)_{n \geq 1}$ via (29) $\tau_{n}^{K}:=\inf \left\{t>0: \sup _{\|\vartheta\|_{\mathbb{R}^{d}} \leq K}|\mu(\vartheta, S(\vartheta, t))| \vee \sup _{\|\vartheta\|_{\mathbb{R}^{d}} \leq K}|\sigma(\vartheta, S(\vartheta, t))| \geq n\right\} \wedge T$.

For all $K>0$, the joint continuity ensures that $\mathbb{P}\left(\tau_{n}^{K}<T\right) \longrightarrow 0$ as $n \longrightarrow \infty$. Let $\theta \in \mathbf{S}\left(\mathbb{R}^{d}\right), \eta \in \mathbf{S}(\mathbb{R})$, and $\max \left\{\eta^{*}, \theta^{*}\right\} \leq K$, where $\eta^{*}=\sup _{t \leq T}\|\eta(t)\|_{\mathbb{R}}$ and $\theta^{*}=\sup _{t \leq T}\|\theta(t)\|_{\mathbb{R}^{d}}$. Without loss of generality, we may and do assume that

$$
\theta(t)=\sum_{i=0}^{n} \theta_{i} \mathbf{1}_{\left(\tau_{i}, \tau_{i+1}\right]}(t) \quad \text { and } \quad \eta(t)=\sum_{i=0}^{n} \eta_{i} \mathbf{1}_{\left(\tau_{i}, \tau_{i+1}\right]}(t)
$$


Denote by $\eta \cdot \int S(\theta, d s)$ the linear stochastic integral of $\eta$ w.r.t. $\int S(\theta, d s)$,

$$
\begin{aligned}
\tilde{\mu}_{u}(\eta, \theta) & :=\sum_{i=0}^{n} \eta_{i} \mu\left(\theta_{i}, S\left(\theta_{i}, \tau_{i}\right)\right) \mathbf{1}_{\left(\tau_{i}, \tau_{i+1}\right]}(u) \quad \text { and } \\
\tilde{\sigma}_{u}(\eta, \theta) & :=\sum_{i=0}^{n} \eta_{i} \sigma\left(\theta_{i}, S\left(\theta_{i}, \tau_{i}\right)\right) \mathbf{1}_{\left(\tau_{i}, \tau_{i+1}\right]}(u) .
\end{aligned}
$$

Then we have

$$
\left(\eta \cdot \int S(\theta, d s)\right)_{t}=\int_{0}^{t} \tilde{\mu}_{u}(\eta, \theta) d A_{u}+\int_{0}^{t} \tilde{\sigma}_{u}(\eta, \theta) d M_{u} .
$$

Moreover, for $C>0$ and $n \geq 1 \in \mathbb{N}$ a straightforward computation leads to

$$
\begin{aligned}
\mathbb{P}\left(\sup _{t \leq T}\left|\left(\eta \cdot \int S(\theta, d s)\right)_{t}\right|>C\right) \leq & \mathbb{P}\left(\sup _{t \leq T}\left|\int_{0}^{\tau_{n}^{K} \wedge t} \tilde{\mu}_{u}(\eta, \theta) d A_{u}\right|>\frac{C}{2}\right) \\
& +\mathbb{P}\left(\sup _{t \leq T}\left|\int_{0}^{\tau_{n}^{K} \wedge t} \tilde{\sigma}_{u}(\eta, \theta) d M_{u}\right|>\frac{C}{2}\right) \\
& +\mathbb{P}\left(\tau_{n}^{K}<T\right) .
\end{aligned}
$$

Thanks to the definition of $\tau_{n}^{K}$, Chebyshev's inequality, and the BurkholderDavis-Gundy inequality, we can conclude that

$$
\begin{aligned}
& \mathbb{P}\left(\sup _{t \leq T}\left|\int_{0}^{\tau_{n}^{K} \wedge t} \tilde{\mu}_{u}(\eta, \theta) d A_{u}\right|>\frac{C}{2}\right) \leq \mathbb{P}\left(A_{T}>\frac{C}{2 n K}\right), \\
& \mathbb{P}\left(\sup _{t \leq T}\left|\int_{0}^{\tau_{n}^{K} \wedge t} \tilde{\sigma}_{u}(\eta, \theta) d M_{u}\right|>\frac{C}{2}\right) \leq \tilde{C} \frac{2 n K}{C} \mathbb{E}\left[\sqrt{[M]_{T}}\right] .
\end{aligned}
$$

Combining these results, we get

$$
\begin{aligned}
\mathbb{P}\left(\sup _{t \leq T}\left|\left(\eta \cdot \int S(\theta, d s)\right)_{t}\right|>C\right) \\
\quad \leq \mathbb{P}\left(\tau_{n}^{K}<T\right)+\mathbb{P}\left(A_{T}>\frac{C}{2 n K}\right)+\tilde{C} \frac{2 n K}{C} \mathbb{E}\left[\sqrt{[M]_{T}}\right] .
\end{aligned}
$$

Since the right-hand side of the inequality is independent of $\theta \in \mathbf{S}\left(\mathbb{R}^{d}\right), \eta \in \mathbf{S}(\mathbb{R})$, and $\mathbb{P}\left(\tau_{n}^{K}<T\right) \longrightarrow 0$ as $n \longrightarrow \infty$, Definition A.8 2 holds (for $C \longrightarrow \infty$ ). Hence, it remains to prove Definition A.8 3. Let $\left(\theta^{n}\right)_{n \geq 1} \subset \mathbf{S}\left(\mathbb{R}^{d}\right)$ be a Cauchy sequence in the $u c p$-topology that is uniformly bounded by $K$ and set $\tau_{m}:=\tau_{m}^{K}$. For $N, n, m \in$ $\mathbb{N}$ we have

$$
\begin{aligned}
d_{\mathcal{S M}} & \left(\int S\left(\theta^{n}, d s\right), \int S\left(\theta^{m}, d s\right)\right) \\
& \leq \mathbb{P}\left(\tau_{N}<T\right)+d_{\mathcal{S M}}\left(\left(\int S\left(\theta^{n}, d s\right)\right)^{\tau_{N}},\left(\int S\left(\theta^{m}, d s\right)\right)^{\tau_{N}}\right) .
\end{aligned}
$$


As $\mathbb{P}\left(\tau_{N}<T\right) \longrightarrow 0$ for $N \longrightarrow \infty$, it remains to show that $\left(\left(\int S\left(\theta^{n}, d s\right)\right)^{\tau_{N}}\right)_{n \geq 1}$ is a Cauchy sequence in the semimartingale topology for all $N \geq 1$. To see this, note that $\left(\left(\theta^{n}\right)^{\tau_{N}}\right)_{n \geq 1}$ is a Cauchy sequence in the $u c p$-topology and Lemma A.15 ensures that for all $N \geq 1$

$$
\left(\int S\left(\theta^{n}, d s\right)\right)^{\tau_{N}}=\int S^{\tau_{N}}\left(\left(\theta^{n}\right)^{\tau_{N}}, d s\right) \quad \forall n \geq 1 .
$$

Due to the joint continuity of $\mu, \sigma, S(\vartheta, t)$, and the dominated convergence theorem for semimartingales ([27], III.13 Théorème), $\left(\int S^{\tau_{N}}\left(\left(\theta^{n}\right)^{\tau_{N}}, d s\right)\right)_{n \geq 1}$ is a Cauchy sequence in the semimartingale topology for all $N \geq 1$.

Proof OF LEMma 4.4. (NA-II) and Theorem 4.2 allow us to write the canonical decompositions of $(S(\vartheta, \cdot))_{\vartheta \in \mathbb{R}^{d}}$ as $S(\vartheta, \cdot)=S(\vartheta, 0)+M^{\vartheta}+\int \lambda^{\vartheta} d\left[M^{\vartheta}\right]$, where $\mathbb{P}\left(\int_{0}^{T}\left(\lambda_{u}^{\vartheta}\right)^{2} d\left[M^{\vartheta}\right]_{u}<\infty\right)=1$ holds for all $\vartheta \in \mathbb{R}^{d}$. For $\left(F\left(\theta^{n}\right)\right)_{n \geq 1} \subset$ $\mathbf{S}\left(\mathbb{R}^{d}\right)$, Theorem 4.2 and Proposition 2.8 ensure that the canonical decompositions of $\left(P^{\theta^{n}}\right)_{n \geq 1}$ are given by

$$
P^{\theta^{n}}=\int S\left(F\left(\theta^{n}\right), d s\right)=S\left(F_{0}\left(\theta^{n}\right), 0\right)+M^{n}+\int \lambda^{(n)} d\left[M^{n}\right],
$$

where $\int_{0}^{T}\left(\lambda_{u}^{(n)}\right)^{2} d\left[M^{n}\right]_{u}<\infty \mathbb{P}$-a.s. for all $n \geq 1$. Besides, for $\left(F\left(\theta^{n}\right)\right)_{n \geq 1}$ with representation (15) we get (17) for all $n \geq 1$. Moreover, 2 and 3 follow from Proposition A.7. Finally, the last statement can be proven along the same lines using Proposition A.14 and Remark 4.1.

ProOF OF THEOREM 4.6. Within the proof, we frequently use the fact that for a Lebesgue-Stieltjes integral process $F=\int H d B$, where $B$ is nondecreasing and right-continuous, its total variation $\operatorname{TV}(F)_{T}$ on the interval $[0, T]$ is $\mathbb{P}$-a.s. given by

$$
\operatorname{TV}(F)_{T}=\int_{0}^{T}\left|H_{u}\right| d B_{u} ;
$$

see, for example, [28], Corollary, page 40. We first prove that there exists a predictable process $\lambda$ such that $A=\int \lambda d[M]$ holds. As $\left[M^{n}\right] \longrightarrow[M]$ in the semimartingale topology, Proposition A.7 ensures that there exists a subsequence (still indexed by $n$ ) such that (recall our convention that equalities between random variables are to be understood $\mathbb{P}$-a.s.)

$$
\begin{array}{r}
\lim _{n \rightarrow \infty} \operatorname{TV}\left(\left[M^{n}\right]-[M]\right)_{T}=0, \\
\lim _{n \rightarrow \infty} \operatorname{TV}\left(\int \lambda^{(n)} d\left[M^{n}\right]-A\right)_{T}=0 .
\end{array}
$$

Therefore, we have

$$
\operatorname{TV}(A)_{T} \leq \liminf _{n \longrightarrow \infty} \operatorname{TV}\left(\int \lambda^{(n)} d\left[M^{n}\right]\right)_{T}
$$


Due to (30), we get for $n \geq 1$

$$
\operatorname{TV}\left(\int \lambda^{(n)} d\left[M^{n}\right]\right)_{T}=\int_{0}^{T}\left|\lambda_{u}^{(n)}\right| d\left[M^{n}\right]_{u}=\int_{0}^{T} 1\left|\lambda_{u}^{(n)}\right| d\left[M^{n}\right]_{u} .
$$

Combining (33) and (34) and applying Hölder's inequality to (34) leads to

$$
\operatorname{TV}(A)_{T} \leq \liminf _{n \rightarrow \infty}\left(\left[M^{n}\right]_{T}\right)^{\frac{1}{2}}\left(\int_{0}^{T}\left(\lambda_{u}^{(n)}\right)^{2} d\left[M^{n}\right]_{u}\right)^{\frac{1}{2}}
$$

According to assumption (19), we can conclude that

$$
\operatorname{TV}(A)_{T} \leq\left([M]_{T}\right)^{\frac{1}{2}} \liminf _{n \rightarrow \infty}\left(\int_{0}^{T}\left(\lambda_{u}^{(n)}\right)^{2} d\left[M^{n}\right]_{u}\right)^{\frac{1}{2}}
$$

Thanks to the Radon-Nikodym theorem, there exists a process $\lambda$ such that

$$
A=\int \lambda d[M]
$$

see [19], 3.13 Proposition, for details. It remains to prove that $\int_{0}^{T} \lambda_{u}^{2} d[M]_{u}<\infty$ holds $\mathbb{P}$-a.s. Due to (31), there exists a set $N^{c}$ of measure zero such that the family $\left(\left[M^{n}\right](\omega)\right)_{n \in \mathbb{N}},[M](\omega)$ is uniformly bounded for all $\omega \in N$. Thanks to Lemma B.1, there exist probability measures $d B(\omega)$ on $[0, T]$ such that

$$
\forall n \in \mathbb{N}: \quad d\left[M^{n}\right](\omega) \ll d B(\omega) \text { and } d[M](\omega) \ll d B(\omega)
$$

hold for all $\omega \in N$, where $N=\Omega \backslash N^{c}$. Due to (30) and (31),

$$
\lim _{n \longrightarrow \infty} \operatorname{TV}\left(\left[M^{n}\right]-[M]\right)_{T}(\omega)=\lim _{n \rightarrow \infty} \int_{0}^{T}\left|\frac{d\left[M^{n}\right]}{d B}-\frac{d[M]}{d B}\right|(\omega, u) d B_{u}(\omega)=0
$$

holds for all $\omega \in N$ and

$$
\frac{d\left[M^{n}\right]}{d B}(\omega, \cdot) \longrightarrow \frac{d[M]}{d B}(\omega, \cdot) \quad \text { in } d B(\omega) \text {-probability. }
$$

Combining (32), (35) and (30), we also have for all $\omega \in N$

$$
\int_{0}^{T}\left|\lambda_{u}^{(n)}(\omega) \frac{d\left[M^{n}\right]}{d B}(\omega, u)-\lambda_{u}(\omega) \frac{d[M]}{d B}(\omega, u)\right| d B_{u}(\omega) \underset{n \longrightarrow \infty}{\longrightarrow} 0
$$

and

$$
\lambda^{(n)}(\omega) \frac{d\left[M^{n}\right]}{d B}(\omega, \cdot) \underset{n \longrightarrow \infty}{\longrightarrow} \lambda .(\omega) \frac{d[M]}{d B}(\omega, \cdot) \quad \text { in } d B(\omega) \text {-probability }
$$

Finally, we may apply Lemma B.3, Fatou's lemma and assumption (19) in order to conclude that the claim is proven. Indeed, for all $\omega \in N$ we have

$$
\begin{aligned}
& \int_{0}^{T} \lambda_{u}^{2}(\omega) d[M]_{u}(\omega) \\
& \quad=\int_{0}^{T} \mathbf{1}_{\left\{\frac{d[M]}{d B} \neq 0\right\}}(\omega, u) \lambda_{u}^{2}(\omega) \frac{d[M]}{d B}(\omega, u) d B_{u}(\omega)
\end{aligned}
$$




$$
\begin{aligned}
& \leq \liminf _{n \longrightarrow \infty} \int_{0}^{T} \mathbf{1}_{\left\{\frac{d[M]}{d B} \neq 0\right\}}(\omega, u)\left(\lambda_{u}^{(n)}(\omega)\right)^{2} \frac{d\left[M^{n}\right]}{d B}(\omega, u) d B_{u}(\omega) \\
& \leq \liminf _{n \longrightarrow \infty} \int_{0}^{T}\left(\lambda_{u}^{(n)}(\omega)\right)^{2} d\left[M^{n}\right]_{u}(\omega) .
\end{aligned}
$$

Proof of Corollary 4.7. As $\left(C_{m}\right)_{m \geq 1} \subset L^{2}(\mathbb{P})$ holds, we know that $\liminf _{n \longrightarrow \infty} \int_{0}^{T}\left(\lambda_{u}^{(n)}\right)^{2} d\left[M^{n}\right]_{u}<\infty$ holds on $\left\{R_{m}=T\right\}$. Since $\mathbb{P}\left(R_{m}=T\right) \longrightarrow 1$ for $m \longrightarrow \infty,(19)$ holds $\mathbb{P}$-a.s.

Proof of Proposition 4.9. Due to [23], Proposition 2.7, the third assumption is equivalent to $\int \lambda^{(n)} d M^{n} \underset{\mathcal{S M}}{\longrightarrow} \int \lambda d M$. According to Proposition A.7, the composition of $C^{2}$-functions with semimartingales is continuous w.r.t. the semimartingale topology. Therefore, we can conclude that

$$
\mathcal{E}\left(-\int \lambda^{(n)} d M^{n}\right) \underset{\mathcal{S M}}{\longrightarrow} \mathcal{E}\left(-\int \lambda d M\right) .
$$

Due to $2, \mathbb{E}\left[\mathcal{E}\left(-\int \lambda d M\right)_{T}\right]=1$ holds and $\mathcal{E}\left(-\int \lambda d M\right)$ is a martingale.

Proof of Lemma 5.3. We set $V_{s}:=V_{s}(\theta)$ and assume w.l.o.g. that $C(\theta) \equiv 0$. Note that it suffices to prove $\mathbb{E}\left[\exp \left(\alpha \sup _{s \leq T}\left|V_{s}\right|\right)\right]<\infty$. This implies that $L^{\theta}$ is a $u$-compatible loss control for $\theta$. As $\theta$ is bounded and $[M]$ is deterministic, we know that for all $\alpha>0$ there exists a constant $K>0$ such that

$$
\begin{aligned}
e^{\alpha \sup _{s \leq T}\left|V_{s}\right|} & \leq \sup _{s \leq T}\left[\exp \left(\alpha V_{s}\right)\right]+\sup _{s \leq T}\left[\exp \left(-\alpha V_{s}\right)\right] \\
& \leq K \sup _{s \leq T} \mathcal{E}\left(-\int \alpha \theta \sigma(\theta) d M\right)_{s}+K \sup _{s \leq T} \mathcal{E}\left(\int \alpha \theta \sigma(\theta) d M\right)_{s} .
\end{aligned}
$$

Due to Novikov's condition, $L^{\theta}$ is a $u$-compatible loss control for $\theta$.

7. Outlook on further research. We would like to take the opportunity to finish this paper with an outlook on further research goals. Introducing the modular perspective to a financial market with a large trader was one major focus of this paper. In particular, we ensured that the approach taken here is a natural extension of the theory on financial markets with small traders. As a result, a rather big portion of the paper was dedicated to the definition of the price process affected by the large trader, Assumption I and its extension to general large trader strategies in Section 4. Consequently, we could only mention other quite interesting aspects in remarks or address them in a rather plain setting.

In the following subsections, we sketch three ideas, highlighting different directions to which we intend to extend our modular approach. While we focus on the question how to include a limit order book into our modular model in the first subsection, the second and third subsection discuss various aspects of arbitrage opportunities for the large trader and different levels of information. The latter is, similar to the analysis in Section 4, closely related to the structure condition (SC). 
7.1. Price process for dynamic strategies and limit order book. In this subsection, we present one approach how to include a limit order book into our modular model. Note that the examples considered so far are models focusing on illiquidity, for example, Bank and Baum [4] and Çetin et al. [10] and price impacts, for example, Almgren-Chriss [2, 3] and Gatheral [16]. In both types of models, the impact of the large trader on her real wealth process and the price process is rather direct. In more recent publications, several authors model the price impact of the large trader via a limit order book and describe the relation between the volume impact and the price impact. Using this approach, we can incorporate a limit order book into our model. While a detailed analysis is left for further research, the next example gives an idea how this can be done.

EXAMPLE 7.1 (Volume impact limit order book model of Alfonsi and Schied [1]). Using the additive compound impact model, we can build a model that is quite similar to the limit order book model of Alfonsi and Schied [1]. In their model, the price impact is driven by the dynamics of a limit order book that is exposed to market orders of the large trader. Our version of the model is characterized as follows. For a strategy,

$$
\theta \in \mathcal{X}:=\{\theta \in b \mathbb{L}(\mathbb{R}) \mid \theta \text { has continuously differentiable paths }\},
$$

the price process is given by

$$
P^{\theta}=\tilde{P}+I^{\theta},
$$

where $\tilde{P}$ is defined as in (7) and the price impact $I^{\theta}$ is given by

$$
I_{t}^{\theta}=H\left(E_{0}\right)+\int_{0}^{t} h\left(E_{u}\right) d E_{u}^{c} .
$$

In the above equation, $h: \mathbb{R} \longrightarrow[0, \infty)$ is an integrable function that is a.s. strictly positive, $H(y)=\int_{0}^{y} h(x) d x$, and $E^{c}$ denotes the continuous part of the volume impact process $E$, whose dynamics are given by

$$
d E_{t}=d \theta_{t}-\rho_{t} E_{t} d t, \quad E_{0} \in \mathbb{R} .
$$

Here, $\rho:[0, \infty) \longrightarrow[0, \infty)$ is a Borel-measurable function reflecting the resilience speed of the volume impact process. Note that for $\theta \in \mathcal{X}$ we get

$$
d E_{t}^{c}=E_{0}\left(-\rho_{t}\right) e^{-\int_{0}^{t} \rho_{v} d v} d t+\left(-\rho_{t} \int_{0}^{t} e^{-\int_{u}^{t} \rho_{v} d v} \dot{\theta}_{u} d u+\dot{\theta}_{t}\right) d t .
$$

Thus, using similar arguments as in Examples 3.4 and 3.5, we can define a proper impact function $F$ ensuring that Assumption I holds and $I^{\theta}$ is given by (36) for all $\theta \in \mathcal{X}$. Instead of presenting these details, we rather elaborate on the connection of this model with the model suggested by Alfonsi and Schied [1]. Here, the choice of the function $h$ is crucial as it links the volume impact on the limit order book to the actual price impact. 
In [1], the actual price process is driven by an unperturbed price $\tilde{P}$ and a response $D$ of the limit order book to market orders $\theta$ of the large trader. This is done in an indirect way by describing the volume impact of the large trader strategy on the limit order book; see (37). In order to connect the volume impact process $E$ to the price impact process $D$, Alfonsi and Schied [1] assume a continuous distribution of the bid and ask spread orders away from the unperturbed price $\tilde{P}$. More precisely, they assume that the distribution is described by a continuous function $g: \mathbb{R} \longrightarrow[0, \infty)$, called the shape function, that is, Lebesgue a.s. strictly positive. Intuitively, the number of shares offered at price $\tilde{P}_{t}+x$ is given by $g(x) d x$. Hence, the volume impact of $E_{t}$ shares is related to the price impact $D_{t}$ via $G\left(D_{t}\right)=E_{t}$, where

$$
G(y):=\int_{0}^{y} g(x) d x, \quad y \in \mathbb{R} .
$$

Since $g$ is continuous and strictly positive, $G$ is invertible and its inverse is

$$
H(y):=G^{-1}(y)=\int_{0}^{y} h(x) d x, \quad y \in \mathbb{R},
$$

where $h(x)=\frac{1}{g(H(x))}$ for $x \in \mathbb{R}$. Summing up, we get

$$
E_{t}=G\left(D_{t}\right) \quad \text { and } \quad D_{t}=H\left(E_{t}\right) .
$$

The connection to our model is provided by the change of variables formula [28], II Section 7, Theorem 31. Indeed, for a predictable càdlàg process $\theta$ of finite variation, whose continuous and purely discontinuous parts are denoted by $\theta^{c}$ and $\theta^{d}$, respectively, we have

$$
D_{t}=H\left(E_{0}\right)+\int_{0}^{t} h\left(E_{u}\right) d E_{u}^{c}+\sum_{u \leq t}\left(H\left(\theta_{u}^{d}\right)-H\left(\theta_{u-}^{d}\right)\right) .
$$

Thus, for $\theta \in \mathcal{X}$ the price impact process $D$ coincides with (36). Finally, we would like to remark that this representation is only a sketch of the model proposed by Alfonsi and Schied [1] for which we refer for details.

7.2. (No) arbitrage opportunities for the large trader. We already fixed our no arbitrage condition for the small trader, namely the no free lunch with vanishing risk condition. Of course, the key question in this subsection is an exact definition of an arbitrage opportunity for the large trader. Following Bank and Baum [4] and the spirit of the fundamental theorem of asset pricing, we could use the classical (NA)-condition. That is, there does not exist an admissible large trader strategy $\theta \in \mathbb{L}(\mathbb{R})$ such that

$$
\mathbb{P}\left(V_{T}(\theta) \geq V_{0}(\theta)\right)=1 \quad \text { and } \quad \mathbb{P}\left(V_{T}(\theta)>V_{0}(\theta)\right)>0 .
$$

Using this notion, Proposition 4.9 provides a tool to check whether or not this condition holds for a tame strategy $\theta \in \mathbb{L}(\mathbb{R})$. The question regarding a proper no arbitrage condition becomes particularly interesting if a limit order book is involved. 
For an analysis of different types of arbitrage opportunities in such a setting, we refer to Alfonsi and Schied [1] and the references therein. Apart from a proper notion for a large trader arbitrage and its dependence on (2), we are interested in the consequences for the small trader once the large trader exploits the arbitrage.

7.3. Different levels of information. In this paper, we always assumed that the small as well as the large trader have the same information modelled by the filtration $\mathbb{F}$. However, it seems to be obvious that different levels of information for the small and the large trader can have substantial impact on the different modules and their analysis. In the following subsection, we discuss some examples that indicate how a weakening of this assumption might influence the analysis of the different modules.

7.3.1. Different levels of information and arbitrage opportunities for the small trader. Consider the illiquid Bachelier model

$$
d P_{t}^{\theta}=\theta_{t} d t+d W_{t},
$$

where $W$ is a standard Brownian motion. Note that this illiquid Bachelier model is our additive compound impact model with impact function $F=i d$. For simplicity, we assume that the cost function is constant zero. Thus, the large trader real wealth process is given by $V(\theta)=\int \theta d P^{\theta}$. For this particular model, it is possible to consider more general large trader strategies which not necessarily have càglàd paths.

In the following, we discuss phenomena arising from different levels of information for the large trader strategy $\theta=-W / t$. Hence, the small trader faces the following price process:

$$
d P_{t}=-\frac{W_{t}}{t} d t+d W_{t}
$$

affected by the large trader strategy $\theta=-W / t$. Moreover, we assume that the filtration (information) $\mathbb{F}^{\mathrm{ST}}$ of the small trader is generated by $P$ defined in (39). Hence, the small trader does not observe the Brownian motion $W$. Besides, the filtration for the large trader $\mathbb{F}^{\mathrm{LT}}$ is generated by $P$ as well as her own strategy. Thus, the large trader does observe $W$.

EXAMPLE 7.2 (Arbitrage opportunities for the small trader that depend on the choice of filtration). For the large trader strategy $\theta=-W / t$, the price process $P^{\theta}$ follows a Brownian motion w.r.t. $\mathbb{F}^{\mathrm{ST}}$; see Jeulin and Yor [22]. Thus, the large trader's action does not lead to arbitrage opportunities for the small trader. This would be different if the small trader would possess the same information as the large trader. Indeed, provided that the small trader's information is given by $\mathbb{F}^{\mathrm{LT}}$, a result by Jeulin and Yor [21] implies

$$
\int_{0}^{\varepsilon} \frac{W_{s}^{2}}{s^{2}} d s=\infty \quad \forall \varepsilon>0 .
$$


Due to Delbaen and Schachermayer [13], this is sufficient for an immediate arbitrage opportunity for the small trader. Note that (40) leads to a failure of the structure condition $(S C)$.

7.3.2. Different levels of information and (no) arbitrage opportunities for the large trader. Questions regarding arbitrage opportunities for the large trader become particularly interesting if she has an edge on information. The next example gives a glimpse of how different levels of information might lead to arbitrage opportunities for the large trader without destabilizing the market and without introducing arbitrage opportunities for the small trader.

EXAMPLE 7.3 (Different levels of information and arbitrage opportunities for the large trader). How can the large trader exploit arbitrage while the small trader cannot? Informally, we can use Example 7.2 and argue as follows: given the information $\mathbb{F}^{\mathrm{ST}}$, the small trader faces a market driven by a $\left(\mathbb{P}, \mathbb{F}^{\mathrm{ST}}\right)$-Brownian motion $P^{\theta}$. Thanks to its martingale property, the small trader wealth process $\int H d P^{\theta}$ is a $\left(\mathbb{P}, \mathbb{F}^{\mathrm{ST}}\right)$-supermartingale whenever $H$ is 1 -admissible. Thus, there are no arbitrage opportunities for the small trader.

In contrast to the small trader, the large trader can exploit her additional knowledge and invest according to $\theta=-W / t$. Intuitively, (40) suggests that this strategy leads to an arbitrage opportunity for the large trader. However, one has to be careful as (40) ensures that $\theta$ is not integrable w.r.t. the Brownian motion $W$. Nevertheless, using arguments similar to those in the proof of [11], Théorème 2.9 ii), the large trader can exploit an unbounded profit with bounded risk without destabilising the market by creating a run away effect in the drift.

\section{APPENDIX A: STRONG NONLINEAR INTEGRATORS AND THE NONLINEAR STOCHASTIC INTEGRAL}

The purpose of Appendix A is to give an overview of strong nonlinear integrators and the nonlinear stochastic integral. For a detailed introduction of these concepts, we refer to Carmona and Nualart [9]. We consider a probability space $(\Omega, \mathcal{F}, \mathbb{P})$ equipped with a filtration $\mathbb{F}=\left(\mathcal{F}_{t}\right)_{t \in[0, T]}$ satisfying the usual conditions. Moreover, $T \in(0, \infty)$ and $\mathcal{F}_{0}$ is trivial apart from null sets. Results from the literature formulated for an infinite time horizon are used by applying the corresponding result to the stopped process.

A.1. Simple strategies. There are various definitions of simple strategies in the literature. Our Definition 2.1 of simple strategies is the one given in Carmona and Nualart [9]. One advantage of this definition is the following unique representation of simple strategies. 
LemmA A.1. Let $\theta \in \mathbf{S}(\mathbb{R})$. Then $\theta_{t+} \in \mathcal{F}_{t}$ for all $t \in[0, T]$. Moreover, define for $t \geq 0$

$$
\Delta^{+} \theta_{t}:=\lim _{h \downarrow 0}\left(\theta_{t+h}-\theta_{t}\right) .
$$

Denote by $t_{1}<\cdots<t_{K}$ the jump times of $\theta \in \mathbf{S}(\mathbb{R})$, that is, those $t \in[0, T]$ such that $\mathbb{P}\left(\Delta^{+} \theta_{t} \neq 0\right)>0$. Besides, set

$$
\Pi:= \begin{cases}\left\{0=t_{0} \leq t_{1}<\cdots<t_{K+1}=T\right\} & \theta \text { jumps with positive probability, } \\ \{0, T\} & \text { else. }\end{cases}
$$

Then

$$
\theta_{-1} \mathbf{1}_{\{0\}}+\sum_{\substack{i=0 \\\left(t_{i}\right) \subset \Pi}}^{K} \theta_{t_{i}+} \mathbf{1}_{\left(t_{i}, t_{i+1}\right]}
$$

is a representation of $\theta$ that is unique in the following sense: $\Pi$ is the smallest deterministic partition of $[0, T]$ that contains $0, T$, and all jump times of $\theta \in \mathbf{S}(\mathbb{R})$ such that the jumps in these points occur with positive probability.

PROOF. Recall that, by assumption, the filtration $\mathbb{F}$ satisfies the usual conditions. Hence, $\mathbb{F}$ is right-continuous. Since $\theta \in \mathbf{S}(\mathbb{R})$ is bounded, the rightcontinuity of $\mathbb{F}$ ensures that $\theta_{t+} \in \mathcal{F}_{t}$ for all $t \in[0, T]$. As $\theta \in \mathbf{S}(\mathbb{R})$, it jumps at most finitely many times. If we define $\Pi$ as above, we know that it contains all jump times of $\theta \in \mathbf{S}(\mathbb{R})$. Hence, (41) is indeed a representation of $\theta$. As $\mathbb{P}\left(\Delta^{+} \theta_{t_{i}} \neq 0\right)>0$ for all $i \in\{1, \ldots, K\}$, uniqueness follows.

The next lemma collects results about càglàd processes. Recall that $f^{*}:=$ $\sup _{t \leq T}\|f(t)\|_{\mathbb{R}^{d}}$ for a bounded, measurable function $f:[0, T] \longrightarrow \mathbb{R}^{d}$.

LEMMA A.2. Let $\left(\theta^{n}\right)_{n \geq 1} \subset \mathbb{L}\left(\mathbb{R}^{d}\right)$ and $\theta \in \mathbb{L}\left(\mathbb{R}^{d}\right)$.

1. If $\theta^{n} \longrightarrow \theta$ in the ucp-topology, then there exists a subsequence (still indexed by $n)$ such that $\left(\theta^{n}-\theta\right)^{*} \longrightarrow 0$ a.s. for $n \longrightarrow \infty$.

2. The sequence $\left(\tau_{m}\right)_{m \geq 1}$ of stopping times, where

$$
\tau_{m}:=\inf \left\{t>0:\left\|\theta_{t}\right\|_{\mathbb{R}^{d}} \geq m\right\} \wedge T,
$$

converges $\mathbb{P}$-a.s. to $T$ and satisfies $\mathbb{P}\left(\tau_{m}<T\right) \longrightarrow 0$ for $m \longrightarrow \infty$.

3. Let $c>0,\left(\theta^{n}\right)_{n \geq 1} \subset \mathbf{S}\left(\mathbb{R}^{d}\right)$, and define for $m \geq 1$ the $i$ th component of $\left(\theta^{m, n}\right)_{n \geq 1} \subset \mathbf{S}^{e}\left(\mathbb{R}^{d}\right)$ by

$$
\left(\theta_{t}^{m, n}\right)^{i}:= \begin{cases}\min \left\{m+c,\left(\theta_{\tau_{m} \wedge t}^{n}\right)^{i}\right\} & \text { on }\left\{\left(\theta^{n}\right)^{i} \geq 0\right\} \\ \max \left\{-m-c,\left(\theta_{\tau_{m} \wedge t}^{n}\right)^{i}\right\} & \text { on }\left\{\left(\theta^{n}\right)^{i}<0\right\}\end{cases}
$$


Then, for all $m \geq 1$, the family $\left\{\left(\theta^{m, n}\right)_{n \geq 1}, \theta^{\tau_{m}}\right\}$ is uniformly bounded by $m+c$. Furthermore, if $\theta^{n} \longrightarrow \theta$ in ucp, then

$$
\theta^{m, n} \stackrel{n \longrightarrow \infty}{u c p} \theta^{\tau_{m}} \quad \text { for all } m \geq 1 .
$$

4. There exists a sequence of extended simple strategies $\left(\theta_{n}\right) \subset \mathbf{S}^{e}\left(\mathbb{R}^{d}\right)$, a sequence $\left(a_{m}\right)_{m \geq 1} \subset \mathbb{N}$, and a sequence of stopping times $\left(\tau_{m}\right)_{m \geq 1} \uparrow T \mathbb{P}$-a.s. such that the following conditions hold:

(a) $\left(\theta_{n}-\theta\right)^{*} \longrightarrow 0 \mathbb{P}$-a.s. for $n \longrightarrow \infty$.

(b) $\mathbb{P}\left(\tau_{m}=T\right) \longrightarrow 1$ for $m \longrightarrow \infty$.

(c) The family $\left\{\left(\theta_{n}^{\tau_{m}}\right)_{n \geq 1}, \theta^{\tau_{m}}\right\}$ is uniformly bounded by $a_{m}$.

PROOF. 1 is well known. In particular, it implies that almost all paths of $\theta \in \mathbb{L}\left(\mathbb{R}^{d}\right)$ are bounded. This fact clearly implies 2 . The first part of 3 holds by definition. As for $m, n \geq 1$ and $0<\epsilon \leq \frac{c}{2}$,

$$
\mathbb{E}\left[1 \wedge\left(\theta^{m, n}-\theta^{\tau_{m}}\right)^{*}\right] \leq \mathbb{P}\left(\left(\theta^{n}-\theta\right)^{*}>\epsilon\right)+\mathbb{E}\left[1 \wedge\left(\theta^{n}-\theta\right)^{*}\right]
$$

holds, the assumption $\theta^{n} \longrightarrow \theta$ in the $u c p$-topology ensures that 3 holds. To prove the last statement, note that Remark 2.2 ensures that there exists a sequence $\left(\tilde{\theta}^{n}\right)_{n \geq 1} \subset \mathbf{S}\left(\mathbb{R}^{d}\right)$ such that $\tilde{\theta}^{n} \longrightarrow \theta$ in the $u c p$-topology. Using the notation of item 3 we define for $c>0$ the sequence $\left(\theta_{n}\right)_{n \geq 1} \subset \mathbf{S}^{e}\left(\mathbb{R}^{d}\right)$ of extended simple strategies by $\theta_{n}:=\tilde{\theta}^{n, n}$. Due to 1 , there exists a subsequence of $\left(\theta_{n}\right)_{n \geq 1} \subset \mathbf{S}^{e}\left(\mathbb{R}^{d}\right)$ that satisfies the desired properties.

Definition A.3. Let $\theta \in \mathbb{L}\left(\mathbb{R}^{d}\right) .\left(\theta^{n}\right)_{n \geq 1} \subset \mathbf{S}^{e}\left(\mathbb{R}^{d}\right)$ is called convenient approximating sequence of $\theta$ if it satisfies all properties of Lemma A.2 4 .

REMARK A.4. The convenient approximating sequences are crucial to prove the existence of the nonlinear stochastic integral for $\theta \in \mathbb{L}\left(\mathbb{R}^{d}\right)$.

A.2. Semimartingale topology. Denote by $\mathbf{S}_{1} \subset \mathbf{S}(\mathbb{R})$ the set of all simple strategies bounded by 1 . Moreover, let $\mathcal{S}$ denote the space of $\mathbb{R}$-valued semimartingales. The semimartingale topology is induced by the metric

$$
\begin{aligned}
& d_{\mathcal{S M}}: \mathcal{S} \times \mathcal{S} \longrightarrow \mathbb{R}_{\geq 0}, \\
&(X, Y) \longmapsto \sup _{H \in \mathbf{S}_{1}} \mathbb{E}\left[1 \wedge\left(\sup _{t \leq T}\left|\int_{0}^{t} H_{u} d(X-Y)_{u}\right|\right)\right] .
\end{aligned}
$$

We say that a sequence $\left(X^{n}\right)_{n \geq 1}$ of semimartingales converges to $X$ in the semimartingale topology if $d_{\mathcal{S M}}\left(X^{n}, X\right) \rightarrow 0$ for $n \longrightarrow \infty$. We also use the notation $X^{n} \underset{\mathcal{S M}}{\longrightarrow}$. 
THEOREM A.5. $\quad\left(\mathcal{S}, d_{\mathcal{S} M}\right)$ is a complete metric space. In particular, the set of continuous semimartingales $\mathcal{S}_{c}$ is a closed subset in $\left(\mathcal{S}, d_{\mathcal{S M}}\right)$, that is, $\left(\mathcal{S}_{c}, d_{\mathcal{S M}}\right)$ is a complete metric space.

ProOF. The first statement is [27], II.7 Théorème. Since convergence in the semimartingale topology implies convergence in the $u c p$-topology, the second statement holds.

Denote by $\mathcal{A}_{\text {loc }}$ the space of all càdlàg adapted processes, whose total variation process is locally integrable. The next result is [27], IV.7 Théorème.

Proposition A.6. The space $\mathcal{A}_{\text {loc }}$ is closed in $\mathcal{S}$. Moreover, for $A \in \mathcal{A}_{\text {loc }}$ we have $d_{\mathcal{S M}}(0, A)=\mathbb{E}\left[1 \wedge \mathrm{TV}(A)_{T}\right]$, where $\mathrm{TV}(A)_{T}$ denotes the total variation of A on $[0, T]$.

The next proposition collects some results on convergence in the semimartingale topology.

Proposition A.7. Let $\left(A^{n}\right)_{n \geq 0}$ be a sequence of processes of finite variation, $\left(M^{n}\right)_{n \geq 0}$ be a sequence of local martingales, and define $S^{n}:=M^{n}+A^{n}$ for $n \geq 0$. If $\left(A^{n}\right)_{n \geq 0} \subset \mathcal{A}_{\text {loc }}$, then $A^{n} \rightarrow A^{0}$ in the semimartingale topology if and only if $\mathrm{TV}\left(A^{n}-A^{0}\right)_{T} \longrightarrow 0$ in probability. Moreover, if $S^{n} \underset{\mathcal{S M}}{\longrightarrow} S^{0}$ then the following statements hold:

1. If $\left(S^{n}\right)_{n \geq 0} \subset \mathcal{S}_{c}$, then $M^{n} \underset{\mathcal{S M}}{\longrightarrow} M^{0}$ and $A^{n} \underset{\mathcal{S M}}{\longrightarrow} A^{0}$.

2. If $f$ is twice continuously differentiable, then $f\left(S^{n}\right) \underset{\mathcal{S M}}{\longrightarrow} f\left(S^{0}\right)$.

3. If $\left(X^{n}\right)_{n \geq 0} \subset \mathcal{S}$ and $X^{n} \underset{\mathcal{S M}}{\longrightarrow} X^{0}$, then $X^{n} S^{n} \underset{\mathcal{S M}}{\longrightarrow} X^{0} S^{0}$.

4. If $\left(S^{n}\right)_{n \geq 0},\left(X^{n}\right)_{n \geq 0} \subset \mathcal{S}_{c}, X^{n} \underset{\mathcal{S M}}{\longrightarrow} X^{0}$, then $\left[X^{n}, S^{n}\right] \underset{\mathcal{S M}}{\longrightarrow}\left[X^{0}, S^{0}\right]$.

PROOF. Without loss of generality, we assume that all processes are a.s. zero in 0. The first statement follows from Proposition A.6. 1 and 2 hold thanks to [27], Remarque IV.3, and [14], Proposition 4. Since

$$
X^{n} S^{n}=\frac{1}{4}\left(\left(X^{n}+S^{n}\right)^{2}-\left(X^{n}-S^{n}\right)^{2}\right)
$$

holds, 2 implies 3 . Thus, it remains to prove 4 Due to the product rule,

$$
\left[X^{n}, S^{n}\right]=X^{n} S^{n}-\int S_{-}^{n} d X^{n}-\int X_{-}^{n} d S^{n}
$$

holds. As convergence in the semimartingale topology implies convergence in the $u c p$-topology, we know that $X_{-}^{n} \rightarrow X_{-}^{0}$ as well as $S_{-}^{n} \rightarrow S_{-}^{0}$ in the $u c p$-topology. Due to 3 and [27], III.13 Théorème, the claim is proven. 
A.3. Strong nonlinear integrators. Here, we give a short overview of strong nonlinear integrators. A detailed discussion can be found in [9]. Throughout this subsection, we consider a family $(S(\vartheta, \cdot))_{\vartheta \in \mathbb{R}^{d}}$ of $\mathbb{R}$-valued, $\mathbb{F}$-adapted, continuous semimartingales on $(\Omega, \mathcal{F}, \mathbb{P})$.

In linear stochastic integration theory, semimartingales build the biggest class of "good integrators". For the nonlinear stochastic integral, the strong nonlinear integrators form the class of "good integrators". In order to give a definition of a strong nonlinear integrator, we need the following notation. Let $L\left(\mathbb{R}^{d}\right)$ denote the set of all deterministic, $\mathbb{R}^{d}$-valued, càglàd functions on the interval $[0, T]$. For $h \in L\left(\mathbb{R}^{d}\right)$ and $t \in(0, T)$ we set $h(t+):=\lim _{t_{n} \downarrow t} h\left(t_{n}\right)$ and $h(t-):=\lim _{t_{n} \uparrow t} h\left(t_{n}\right)$. Furthermore, the elementary nonlinear stochastic integral of $\theta \in \mathbf{S}\left(\mathbb{R}^{d}\right)$ w.r.t. $(S(\vartheta, \cdot))_{\vartheta \in \mathbb{R}^{d}}$, where the representation of $\theta \in \mathbf{S}\left(\mathbb{R}^{d}\right)$ is given in (3), is defined as

$$
\int_{0}^{t} S\left(\theta_{s}, d s\right):=S\left(\theta_{-1}, 0\right)+\sum_{i=0}^{n}\left\{S\left(\theta_{\tau_{i} \wedge t+}, \tau_{i+1} \wedge t\right)-S\left(\theta_{\tau_{i} \wedge t+}, \tau_{i} \wedge t\right)\right\}
$$

Definition A.8 ([9], Proposition II.3.1). $\quad(S(\vartheta, \cdot))_{\vartheta \in \mathbb{R}^{d}}$ is a strong non-linear integrator if the following conditions hold:

1. For all $t \in(0, T]$ and all $h, h^{\prime} \in L\left(\mathbb{R}^{d}\right)$ we have $S(h, t)=S\left(h^{\prime}, t\right)$ outside of a $\mathbb{P}$-null set (possibly depending upon $h, h^{\prime}$ and $t$ ) whenever

$$
h(s)=h^{\prime}(s) \quad \text { for all } s \leq t \quad \text { and } \quad h(t+)=h^{\prime}(t+) .
$$

2. For all fixed $t \in(0, T]$ and $K>0$, the set of random variables $(\eta \times$ $\left.\int S(\theta, d s)\right)_{t}$ with $\theta \in \mathbf{S}\left(\mathbb{R}^{d}\right), \eta \in \mathbf{S}$ and $\max \left\{\theta^{*}, \eta^{*}\right\} \leq K$ is bounded in probability.

3. For fixed $t<T$, the mapping $\theta \longmapsto \int S^{t}(\theta, d s)$ is locally uniformly continuous from $\mathbf{S}\left(\mathbb{R}^{d}\right)$, endowed with the $u c p$-topology, into $\left(\mathcal{S}, d_{\mathcal{S M}}\right)$.

REMARK A.9. This definition is one way to define strong nonlinear integrators; see [9], Proposition II.3.1. For our purposes, 3 is important. It allows us to define the nonlinear stochastic integral for càglàd-processes.

Note that Definition A.8 1 . is a regularity property of $(S(\vartheta, \cdot))_{\vartheta \in \mathbb{R}^{d}}$. The next lemma provides sufficient conditions ensuring that $(S(\vartheta, \cdot))_{\vartheta \in \mathbb{R}^{d}}$ satisfies Definition A.8 1.

LEMMA A.10. Let $d=1 .(S(\vartheta, \cdot))_{\vartheta \in \mathbb{R}}$ satisfies the first item of Definition A.8 if at least one of the following conditions hold for almost all $\omega \in \Omega$ :

1. The mapping $S(\cdot, \cdot, \omega): \mathbb{R} \times[0, T] \longrightarrow \mathbb{R}$ is jointly continuous.

2. $S$ is continuous in both arguments and satisfies $(\mathrm{O})$; see page 3745. 
PROOF. The first statement is clear. To prove the second statement, we show that Condition (O) implies joint continuity. We oppress the $\omega$ in the following proof. Let $(\bar{\vartheta}, \bar{t}) \in \mathbb{R} \times[0, T]$. Since $S(\cdot, \bar{t})$ is continuous, for all $\epsilon>0$ we can choose an open interval $\left(\vartheta_{\min }, \vartheta_{\max }\right) \ni \bar{\vartheta}$ such that

$$
\left|S\left(\vartheta_{\min }, \bar{t}\right)-S\left(\vartheta_{\max }, \bar{t}\right)\right|<\frac{\epsilon}{3} \text {. }
$$

Besides, $S\left(\vartheta_{\min }, \cdot\right)$ and $S\left(\vartheta_{\max }, \cdot\right)$ are continuous, too. Therefore, for all $\epsilon>0$ we can find $\delta>0$ such that for all $t \in(\bar{t}-\delta, \bar{t}+\delta) \cap[0, T]$

$$
\left|S\left(\vartheta_{\min }, t\right)-S\left(\vartheta_{\min }, \bar{t}\right)\right|<\frac{\epsilon}{3} \quad \text { as well as }\left|S\left(\vartheta_{\max }, t\right)-S\left(\vartheta_{\max }, \bar{t}\right)\right|<\frac{\epsilon}{3} \text {. }
$$

Thanks to the triangle inequality, we get

$$
|S(\bar{\vartheta}, \bar{t})-S(\vartheta, t)| \leq|S(\bar{\vartheta}, \bar{t})-S(\vartheta, \bar{t})|+|S(\vartheta, \bar{t})-S(\vartheta, t)|
$$

for $(\vartheta, t) \in\left(\vartheta_{\min }, \vartheta_{\max }\right) \times(\bar{t}-\delta, \bar{t}+\delta) \cap[0, T]$. Due to (43) and the monotonicity of $S(\cdot, \bar{t})$, the first term in the above equation is less than $\frac{\epsilon}{3}$ for all $\vartheta \in\left(\vartheta_{\min }, \vartheta_{\max }\right)$. By monotonicity, the second term is less than $\frac{2 \epsilon}{3}$ for all $\vartheta \in\left(\vartheta_{\min }, \vartheta_{\max }\right)$ and for all $t \in(\bar{t}-\delta, \bar{t}+\delta) \cap[0, T]$. Indeed, suppose

$$
|S(\vartheta, t)-S(\vartheta, \bar{t})|=S(\vartheta, t)-S(\vartheta, \bar{t}) .
$$

Due to monotonicity, we get

$$
\begin{aligned}
-\frac{2 \epsilon}{3} & \leq S\left(\vartheta_{\min }, t\right)-S\left(\vartheta_{\min }, \bar{t}\right)+S\left(\vartheta_{\min }, \bar{t}\right)-S\left(\vartheta_{\max }, \bar{t}\right) \\
& \leq S(\vartheta, t)-S(\vartheta, \bar{t}) \\
& \leq S\left(\vartheta_{\max }, t\right)-S\left(\vartheta_{\max }, \bar{t}\right)+S\left(\vartheta_{\max }, \bar{t}\right)-S\left(\vartheta_{\min }, \bar{t}\right) \leq \frac{2 \epsilon}{3}
\end{aligned}
$$

A.4. Nonlinear stochastic integral. Let $\theta \in \mathbf{S}\left(\mathbb{R}^{d}\right)$ with representation (3). In [9], the elementary nonlinear stochastic integral of $\theta$ w.r.t. a strong nonlinear integrator $(S(\vartheta, \cdot))_{\vartheta \in \mathbb{R}^{d}}$ is defined by (42). Moreover, the definition of a strong nonlinear integrator allows us to define the nonlinear stochastic integral of $\theta \in$ $b \mathbb{L}\left(\mathbb{R}^{d}\right)$ w.r.t. $(S(\vartheta, \cdot))_{\vartheta \in \mathbb{R}^{d}}$ as the limit in the semimartingale topology.

DEFINITION A.11. Let $(S(\vartheta, \cdot))_{\vartheta \in \mathbb{R}^{d}}$ be a strong nonlinear integrator, $\theta \in$ $b \mathbb{L}\left(\mathbb{R}^{d}\right)$, and let $\left(\theta^{n}\right)_{n \geq 1} \subset \mathbf{S}\left(\mathbb{R}^{d}\right)$ denote a uniformly bounded sequence of simple strategies such that $\theta^{n} \rightarrow \theta$ in the ucp-topology. The nonlinear stochastic integral $\int S(\theta, d s)$ of $\theta \in b \mathbb{L}\left(\mathbb{R}^{d}\right)$ w.r.t. $(S(\vartheta, \cdot))_{\vartheta \in \mathbb{R}^{d}}$ is the limit of $\left(\int S\left(\theta^{n}, d s\right)\right)_{n \geq 1}$ in the semimartingale topology, that is,

$$
\int S\left(\theta^{n}, d s\right) \underset{\mathcal{S M}}{\longrightarrow} \int S(\theta, d s)
$$


REMARK A.12. 1. Since $(S(\vartheta, \cdot))_{\vartheta \in \mathbb{R}^{d}}$ is a strong nonlinear integrator, the limit in the above definition exists.

2. Let $d=1$. If $S(\vartheta, \cdot)=\vartheta S(0, \cdot)$ for all $\vartheta \in \mathbb{R}$, the definition coincides with the classical linear stochastic integral.

A diagonalization argument and Definition A.11 lead to the next lemma.

LEMMA A.13. Let $(S(\vartheta, \cdot))_{\vartheta \in \mathbb{R}^{d}}$ be a strong nonlinear integrator, $\theta \in$ $b \mathbb{L}\left(\mathbb{R}^{d}\right)$, and let $\left(\theta^{n}\right)_{n \geq 1} \subset b \mathbb{L}\left(\mathbb{R}^{d}\right)$ denote a uniformly bounded sequence of strategies such that $\theta^{n} \rightarrow \theta$ in the ucp-topology. Then $\left(\int S\left(\theta^{n}, d s\right)\right)_{n \geq 1}$ is a Cauchy sequence in the semimartingale topology and

$$
\int S\left(\theta^{n}, d s\right) \underset{\mathcal{S M}}{\longrightarrow} \int S(\theta, d s)
$$

The next proposition provides an explicit expression for the nonlinear stochastic integral of an extended simple strategy. let

PROPOSITION A.14. Let $(S(\vartheta, \cdot))_{\vartheta \in \mathbb{R}^{d}}$ be a strong nonlinear integrator and

$$
\theta(t)=\theta_{-1} \mathbf{1}_{\{0\}}+\sum_{i=0}^{n} \theta_{i} \mathbf{1}_{\left(\tau_{i}, \tau_{i+1}\right]}(t)
$$

be an $\mathbb{R}^{d}$-valued extended simple strategy. Then $\int S(\theta, d s)$ is given by

$$
\int_{0}^{t} S\left(\theta_{s}, d s\right)=S\left(\theta_{-1}, 0\right)+\sum_{i=0}^{n}\left\{S\left(\theta_{\tau_{i} \wedge t+}, \tau_{i+1} \wedge t\right)-S\left(\theta_{\tau_{i} \wedge t+}, \tau_{i} \wedge t\right)\right\} .
$$

PROOF. To prove the statement, we construct a uniformly bounded sequence of simple strategies $\left(\theta^{m}\right)_{m \geq 1} \subset \mathbf{S}\left(\mathbb{R}^{d}\right)$ that converges to $\theta$ in the $u c p$-topology. Define for $i \in\{0, \ldots, n\}$ the sequence $\left(\tau_{i}^{m}\right)_{m \geq 1}$ via

$$
\tau_{i}^{m}:=\frac{\left\lfloor 2^{m} \tau_{i}\right\rfloor+1}{2^{m}} .
$$

By definition, $\tau_{i}^{m} \downarrow \tau_{i}$ a.s. for $i \in\{0, \ldots, n\}$. Furthermore, $\left(\tau_{i}^{m}\right)_{m \geq 1}$ is a sequence of stopping times. Indeed, let $k \geq 0$ such that $r \in\left[\frac{k}{2^{m}}, \frac{k+1}{2^{m}}\right) \subset[0, T)$ holds. Then

$$
\left\{\tau_{i}^{m}<r\right\}=\left\{\tau_{i}<\frac{k}{2^{m}}\right\} \in \mathcal{F}_{r} .
$$

Since, by assumption, the filtration is right-continuous, we have for $t \in[0, T)$

$$
\left\{\tau_{i}^{m} \leq t\right\}=\bigcap_{r>t}\left\{\tau_{i}^{m}<r\right\} \in \mathcal{F}_{t} .
$$


Define $\left(\theta^{m}\right)_{m \geq 1} \subset \mathbf{S}\left(\mathbb{R}^{d}\right)$ via

$$
\theta^{m}(t)=\theta_{-1} \mathbf{1}_{\{0\}}+\sum_{i=0}^{n} \theta_{i} \mathbf{1}_{\left(\tau_{i}^{m}, \tau_{i+1}^{m}\right]}(t) .
$$

By definition $\theta^{m} \longrightarrow \theta$ in the $u c p$-topology. Since $S$ is a strong nonlinear integrator, the sequence $\int S\left(\theta^{m}, d s\right)$ converges to $\int S(\theta, d s)$ in the semimartingale topology. Due to the fact that the limit in the semimartingale topology and the limit in the $u c p$-topology are indistinguishable, the following computation proves the statement:

$$
\begin{aligned}
\int_{0}^{t} S\left(\theta_{s}^{m}, d s\right)-S\left(\theta_{-1}, 0\right) & =\sum_{i=0}^{n}\left\{S\left(\theta_{\tau_{i}^{m} \wedge t+}, \tau_{i+1}^{m} \wedge t\right)-S\left(\theta_{\tau_{i}^{m} \wedge t+}, \tau_{i}^{m} \wedge t\right)\right\} \\
& =\sum_{i=0}^{n}\left\{S\left(\theta_{i}, \tau_{i+1}^{m} \wedge t\right)-S\left(\theta_{i}, \tau_{i}^{m} \wedge t\right)\right\} \\
& \underset{u c p}{\longrightarrow} \sum_{i=0}^{n}\left\{S\left(\theta_{i}, \tau_{i+1} \wedge t\right)-S\left(\theta_{i}, \tau_{i} \wedge t\right)\right\} \\
& =\sum_{i=0}^{n}\left\{S\left(\theta_{\tau_{i} \wedge t+}, \tau_{i+1} \wedge t\right)-S\left(\theta_{\tau_{i} \wedge t+}, \tau_{i} \wedge t\right)\right\} .
\end{aligned}
$$

Next, we need rules for stopping times and nonlinear stochastic integrals.

LEMMA A.15. Let $(S(\vartheta, \cdot))_{\vartheta \in \mathbb{R}^{d}}$ be a strong nonlinear integrator, let $\tau$ be a stopping time, and $\theta \in b \mathbb{L}\left(\mathbb{R}^{d}\right)$. Then

$$
\begin{aligned}
\int_{0}^{\tau \wedge t} S\left(\theta_{s}, d s\right) & =\int_{0}^{t} S^{\tau}\left(\theta_{s}, d s\right)=\int_{0}^{t} S^{\tau}\left(\theta_{s}^{\tau}, d s\right) \\
& =\int_{0}^{\tau \wedge t} S\left(\theta_{s}^{\tau}, d s\right)=\int_{0}^{\tau \wedge t} S^{\tau}\left(\theta_{s}^{\tau}, d s\right) .
\end{aligned}
$$

Proof. Due to Proposition A.14, it is straightforward to check that the statement holds for all extended simple strategies. Since $(S(\vartheta, \cdot))_{\vartheta \in \mathbb{R}^{d}}$ is a strong nonlinear integrator, the result holds for $\theta \in b \mathbb{L}\left(\mathbb{R}^{d}\right)$ by an approximation argument.

Now, we are able to prove the existence of nonlinear stochastic integrals for $\theta \in \mathbb{L}\left(\mathbb{R}^{d}\right)$.

THEOREM A.16. Let $(S(\vartheta, \cdot))_{\vartheta \in \mathbb{R}^{d}}$ be a strong nonlinear integrator and $\theta \in$ $\mathbb{L}\left(\mathbb{R}^{d}\right)$. Moreover, let $\left(\theta^{n}\right)_{n \geq 1} \subset \mathbf{S}\left(\mathbb{R}^{d}\right)$ such that $\theta^{n} \rightarrow \theta$ in the ucp-topology. Then 
$\left(\int S\left(\theta^{n}, d s\right)\right)_{n \geq 1}$ is a Cauchy sequence in the semimartingale topology. Besides, there exists a semimartingale $\int S(\theta, d s)$ such that

$$
\int S\left(\theta^{n}, d s\right) \underset{\mathcal{S M}}{\longrightarrow} \int S(\theta, d s) .
$$

Furthermore, $\int S(\theta, d s)$ is independent of the approximating sequence.

PROOF. We have to prove that $\left(\int S\left(\theta^{n}, d s\right)\right)_{n \geq 1}$ is a Cauchy sequence in the semimartingale topology. Then Theorem A.5 ensures that there exists a limit, denoted by $\int S(\theta, d s)$, such that (44) holds. Due to Definition A.8 3, the limit does not depend on the approximating sequence. Let $N, n, m \geq 1, c>0$ and recall the notation of Lemma A.2. As $\theta^{n}=\theta^{N, n}$ and $\theta^{m}=\theta^{N, m}$ on

$$
\left\{\tau_{N}=T\right\} \cap\left\{\sup _{s \leq T}\left\|\theta_{s}^{n}-\theta_{s}^{m}\right\|_{\mathbb{R}^{d}} \leq \frac{c}{2}\right\},
$$

Proposition A.14 guarantees that on this set

$$
\int S\left(\theta^{n}, d s\right)=\int S\left(\theta^{N, n}, d s\right) \text { and } \int S\left(\theta^{m}, d s\right)=\int S\left(\theta^{N, m}, d s\right)
$$

holds. Hence, we get

$$
\begin{aligned}
d_{\mathcal{S M}}\left(\int S\left(\theta^{n}, d s\right), \int S\left(\theta^{m}, d s\right)\right) \\
\leq \mathbb{P}\left(\tau_{N}<T\right)+\mathbb{P}\left(\left(\theta^{n}-\theta^{m}\right)^{*}>\frac{c}{2}\right) \\
\quad+d_{\mathcal{S M}}\left(\int S\left(\theta^{N, n}, d s\right), \int S\left(\theta^{N, m}, d s\right)\right) .
\end{aligned}
$$

As $\left(\theta^{n}\right)_{n \geq 1} \subset \mathbf{S}\left(\mathbb{R}^{d}\right)$ is a Cauchy sequence in the $u c p$-topology, Lemma A.2 and Lemma A.13 ensure that the sequence $\left(\int S\left(\theta^{n}, d s\right)\right)_{n \geq 1}$ is a Cauchy sequence in the semimartingale topology for all $N \geq 1$.

DEFINITION A.17. Let $(S(\vartheta, \cdot))_{\vartheta \in \mathbb{R}^{d}}$ be a strong nonlinear integrator, $\theta \in$ $\mathbb{L}\left(\mathbb{R}^{d}\right)$ and $\left(\theta^{n}\right)_{n \geq 1} \subset \mathbf{S}\left(\mathbb{R}^{d}\right)$ such that $\theta^{n} \rightarrow \theta$ in the $u c p$-topology. The semimartingale $\int S(\theta, d s)$ in Theorem A.16 is called the nonlinear stochastic integral of $\theta$ w.r.t. $(S(\vartheta, \cdot))_{\vartheta \in \mathbb{R}^{d}}$.

\section{APPENDIX B: MISCELLANEOUS RESULTS}

LemmA B.1. Let $\left(\mu_{n}\right)_{n \geq 1}$ be bounded measures on $(\Omega, \mathcal{F})$. There exists a probability measure $\mathbb{P}$ on $(\Omega, \mathcal{F})$ such that $\mu_{n} \ll \mathbb{P}$ holds for all $n \in \mathbb{N}$. 
PROOF. As all measures dominate the zero-measure, we assume w.l.o.g. that $\mu_{n}(\Omega)>0$ for all $n \in \mathbb{N}$. Define $\tilde{\mu}_{n}:=\frac{1}{\mu_{n}(\Omega)} \mu_{n}$ for $n \in \mathbb{N}$. As $\tilde{\mu}_{n} \sim \mu_{n}$ for all $n \in \mathbb{N}$, it suffices to prove the lemma for the probability measures $\left(\tilde{\mu}_{n}\right)_{n \geq 1}$. Choose $\left(\alpha_{n}\right)_{n \geq 1} \subset \mathbb{R}$ such that $\alpha_{n}>0$ for all $n \in \mathbb{N}$ and $\sum_{n=1}^{\infty} \alpha_{n}=1$ holds. Then the measure $\mathbb{P}:=\sum_{n=1}^{\infty} \alpha_{n} \tilde{\mu}_{n}$ satisfies the desired properties.

THEOREM B.2 (Continuous mapping theorem). Let $\left(M_{1}, d_{1}\right),\left(M_{2}, d_{2}\right)$ be complete metric spaces and $(\Omega, \mathcal{F}, \mathbb{P})$ be a probability space. Further, let

$$
\begin{aligned}
X, X_{n}:(\Omega, \mathcal{F}, \mathbb{P}) & \longrightarrow\left(M_{1}, d_{1}\right), \quad n \in \mathbb{N}, \\
h:\left(M_{1}, d_{1}\right) & \longrightarrow\left(M_{2}, d_{2}\right)
\end{aligned}
$$

be measurable functions such that $\mathbb{P}\left(X \in D_{h}\right)=0$, where $D_{h} \subset M_{1}$ denotes the set of discontinuity points of $h$. Then the following holds:

$$
X_{n} \longrightarrow X \quad \text { in } \mathbb{P} \text {-probability } \quad \Longrightarrow \quad h\left(X_{n}\right) \longrightarrow h(X) \quad \text { in } \mathbb{P} \text {-probability. }
$$

PROOF. The proof of [34], 2.3 Theorem, translates to this setting.

LEMmA B.3. For all $n \in \mathbb{N}$ let $g_{n}, g, f_{n}, f$ be measurable functions on the probability space $(\Omega, \mathcal{F}, \mathbb{P})$. Further, let $g_{n}, g \geq 0 \mathbb{P}$-a.s. for all $n \in \mathbb{N}$,

$f_{n} g_{n} \longrightarrow f g \quad$ in $\mathbb{P}$-probability and $g_{n} \longrightarrow g \quad$ in $\mathbb{P}$-probability.

Then

$$
\mathbf{1}_{\{g \neq 0\}} f_{n}^{2} g_{n} \longrightarrow \mathbf{1}_{\{g \neq 0\}} f^{2} g \quad \text { in } \mathbb{P} \text {-probability. }
$$

PROOF. Due to Theorem B.2 and the assumptions, we have

$$
f_{n}^{2} g_{n}^{2} \longrightarrow f^{2} g^{2} \quad \text { and } \quad \frac{1}{g_{n}+\mathbf{1}_{\{g=0\}}} \longrightarrow \frac{1}{g+\mathbf{1}_{\{g=0\}}}
$$

in $\mathbb{P}$-probability. Applying Theorem B.2 again, we get

$$
\frac{f_{n}^{2} g_{n}^{2}}{g_{n}+\mathbf{1}_{\{g=0\}}} \longrightarrow \frac{f^{2} g^{2}}{g+\mathbf{1}_{\{g=0\}}} \quad \text { in } \mathbb{P} \text {-probability. }
$$

This implies the desired convergence in $\mathbb{P}$-probability.

Acknowledgements. We would like to thank Jan Kallsen for inspiring discussions and his support for this project. In particular, Jan was involved in the original construction of the example about exponential utility maximization. Moreover, we are grateful for his hospitality at research visits and his careful reading of the final version. 


\section{REFERENCES}

[1] Alfonsi, A. and Schied, A. (2010). Optimal trade execution and absence of price manipulations in limit order book models. SIAM J. Financial Math. 1 490-522. MR2669402

[2] Almgren, R. and Chriss, N. (1999). Value under liquidation. Risk 12 61-63.

[3] Almgren, R. and Chriss, N. (2000). Optimal execution of portfolio transactions. J. Risk 3 5-39.

[4] BANK, P. and BAUM, D. (2004). Hedging and portfolio optimization in financial markets with a large trader. Math. Finance $141-18$. MR2030833

[5] Biagini, S. and Frittelli, M. (2005). Utility maximization in incomplete markets for unbounded processes. Finance Stoch. 9 493-517. MR2212892

[6] Biagini, S. and Frittelli, M. (2008). A unified framework for utility maximization problems: An Orlicz space approach. Ann. Appl. Probab. 18 929-966. MR2418234

[7] Biagini, S. and Sîrbu, M. (2012). A note on admissibility when the credit line is infinite. Stochastics 84 157-169. MR2916872

[8] BogacheV, V. I. (2007). Measure Theory, Vols. I, II. Springer, Berlin. MR2267655

[9] Carmona, R. A. and Nualart, D. (1990). Nonlinear Stochastic Integrators, Equations and Flows. Stochastics Monographs 6. Gordon \& Breach Science Publishers, New York. MR1056476

[10] Çetin, U., JARrow, R. A. and Protter, P. (2004). Liquidity risk and arbitrage pricing theory. Finance Stoch. 8 311-341. MR2213255

[11] Choulli, T. and STRicker, C. (1996). Deux applications de la décomposition de GaltchoukKunita-Watanabe. In Séminaire de Probabilités, XXX. (J. Azéma, M. Émery and M. Yor, eds.). Lecture Notes in Math. 1626 12-23. Springer, Berlin. MR1459473

[12] Delbaen, F. and Schachermayer, W. (1994). A general version of the fundamental theorem of asset pricing. Math. Ann. 300 463-520. MR1304434

[13] Delbaen, F. and Schachermayer, W. (1995). The existence of absolutely continuous local martingale measures. Ann. Appl. Probab. 5 926-945. MR1384360

[14] Emery, M. (1979). Une topologie sur l'espace des semimartingales. In Séminaire de Probabilités, XIII (Univ. Strasbourg, Strasbourg, 1977/78). Lecture Notes in Math. 721 260-280. Springer, Berlin. MR0544800

[15] Frey, R. (1998). Perfect option hedging for a large trader. Finance Stoch. 2 115-141.

[16] Gatheral, J. (2010). No-dynamic-arbitrage and market impact. Quant. Finance 10 749-759. MR2741947

[17] Gatheral, J. and Schied, A. (2013). Dynamical models of market impact and algorithms for order execution. In Handbook on Systemic Risk (J. P. Fouque and J. A. Langsam, eds.) 579-602. Cambridge Univ. Press, Cambridge.

[18] Hulley, H. and SchWeizer, M. (2010). $\mathrm{M}^{6}$ —On minimal market models and minimal martingale measures. In Contemporary Quantitative Finance (C. Chiarella and A. Novikov, eds.) 35-51. Springer, Berlin. MR2732839

[19] JACOD, J. and ShIRYAeV, A. N. (2003). Limit Theorems for Stochastic Processes, 2nd ed. Grundlehren der Mathematischen Wissenschaften [Fundamental Principles of Mathematical Sciences] 288. Springer, Berlin. MR1943877

[20] Jarrow, R. (1992). Market manipulation, bubbles, corners, and short squeezes. J. Financ. Quant. Anal. 27 311-336.

[21] Jeulin, T. and Yor, M. (1979). Inégalité de Hardy, semimartingales, et faux-amis. In Séminaire de Probabilités, XIII (Univ. Strasbourg, Strasbourg, 1977/78). Lecture Notes in Math. 721 332-359. Springer, Berlin. MR0544805

[22] Jeulin, T. and Yor, M. (1990). Filtration des ponts browniens et équations différentielles stochastiques linéaires. In Séminaire de Probabilités, XXIV, 1988/89. Lecture Notes in Math. 1426 227-265. Springer, Berlin. MR1071543 
[23] Kardaras, C. (2013). On the closure in the Emery topology of semimartingale wealthprocess sets. Ann. Appl. Probab. 23 1355-1376. MR3098435

[24] KÜHN, C. (2006). Optimal investment in financial markets with different liquidity effects. Preprint, MathFinance Institute, Goethe Univ., Frankfurt, Germany.

[25] Kunita, H. (1997). Stochastic Flows and Stochastic Differential Equations. Cambridge Studies in Advanced Mathematics 24. Cambridge Univ. Press, Cambridge. Reprint of the 1990 original. MR1472487

[26] Kyle, A. (1985). Continuous auctions and insider trading. Econometrica 53 1315-1335.

[27] MÉmin, J. (1980). Espaces de semi martingales et changement de probabilité. Z. Wahrsch. Verw. Gebiete 52 9-39. MR0568256

[28] Protter, P. E. (2005). Stochastic Integration and Differential Equations, 2nd ed. Stochastic Modelling and Applied Probability 21. Springer, Berlin. Version 2.1, corrected third printing. MR2273672

[29] Revuz, D. and Yor, M. (1999). Continuous Martingales and Brownian Motion, 3rd ed. Grundlehren der Mathematischen Wissenschaften [Fundamental Principles of Mathematical Sciences] 293. Springer, Berlin. MR1725357

[30] Schachermayer, W. (1993). A counterexample to several problems in the theory of asset pricing. Math. Finance 3 217-229.

[31] SCHIED, A. (2013). Robust strategies for optimal order execution in the Almgren-Chriss framework. Appl. Math. Finance 20 264-286. MR3169854

[32] Schied, A. and Schöneborn, T. (2009). Risk aversion and the dynamics of optimal liquidation strategies in illiquid markets. Finance Stoch. 13 181-204. MR2482051

[33] Schied, A., Schöneborn, T. and Tehranchi, M. (2010). Optimal basket liquidation for CARA investors is deterministic. Appl. Math. Finance 17 471-489. MR2786971

[34] VAn DeR VAarT, A. W. (1998). Asymptotic Statistics. Cambridge Series in Statistical and Probabilistic Mathematics 3. Cambridge Univ. Press, Cambridge. MR1652247

DEPARTMENT OF MATHEMATICS

UNIVERSITY OF VIENNA

OSKAR-MORGENSTERN-PLATZ 1

1090 VIENNA

AUSTRIA

E-MAIL: tilmann.bluemmel@univie.ac.at
Financial AND ACtUARIAl Mathematics VIENNA UNIVERSITY OF TECHNOLOGY

WIEDNER HAUPTSTRASSE 8-10

1040 VIENNA

AUSTRIA

E-MAIL: rheinlan@fam.tuwien.ac.at

URL: https://fam.tuwien.ac.at/ rheinlan 